\title{
Cluster Synchronization Control for Discrete-Time Complex Dynamical Networks: When Data Transmission Meets Constrained Bit Rate
}

\author{
Jun-Yi Li, Zidong Wang, Renquan $\mathrm{Lu}$ and Yong $\mathrm{Xu}$
}

\begin{abstract}
In this paper, the cluster synchronization control problem is studied for discrete-time complex dynamical networks when the data transmission is subject to constrained bit rate. A bit rate model is presented to quantify the limited network bandwidth, and the effects from the constrained bit rate onto the control performance of the cluster synchronization are evaluated. A sufficient condition is first proposed to guarantee the ultimate boundedness of the error dynamics of the cluster synchronization, and then a bit rate condition is established to reveal the fundamental relationship between the bit rate and certain performance index of the cluster synchronization. Subsequently, two optimization problems are formulated to design the desired synchronization controllers with aim to achieve two distinct synchronization performance indices. The co-design issue for the bit rate allocation protocol and the controller gains is further discussed to reduce the conservatism by locally minimizing a certain asymptotic upper bound of the synchronization error dynamics. Finally, three illustrative simulation examples are utilized to validate the feasibility and effectiveness of the developed synchronization control scheme.
\end{abstract}

Index Terms-Cluster synchronization control, constrained bit rate, coding-decoding, ultimate boundedness, co-design problem.

\section{INTRODUCTION}

Complex dynamical networks (CDNs), which are composed of a large number of highly interconnected dynamical units, can be used to describe many real-world dynamical systems including, but are not limited to, neural networks, social relationship networks, coupled biological/chemical systems, and World Wide Webs [2]. With the ever-increasing demands of understanding the dynamical characteristics of a large number of real-world networks, the CDNs have received much research attention from many disciplines such as physics, nonlinear science, mathematics, and computer and information science [8], [9], [11], [16], [22], [33], [34], [52].

This work was supported in part by the Local Innovative and Research Teams Project of Guangdong Special Support Program of China under grant 2019BT02X353, the Innovative Research Team Program of Guangdong Province Science Foundation of China under grant 2018B030312006, the National Key Research and Development Program of China under grant 2018YFB1700400, the National Natural Science Foundation of China under Grants 61873148 and 61933007, and the Alexander von Humboldt Foundation of Germany. (Corresponding author: Renquan Lu.)

J.-Y. Li, R. Lu and Y. Xu are with the School of Automation and Guangdong Province Key Laboratory of Intelligent Decision and Cooperative Control, Guangdong University of Technology, Guangzhou 510006, China (e-mails: jun-yi-li@foxmail.com,rqlu@gdut.edu.cn, xuyong809@163.com).

Z. Wang is with the Department of Computer Science, Brunel University London, Uxbridge, Middlesex, UB8 3PH, United Kingdom. (e-mail: zidong.wang@brunel.ac.uk)
As a collective feature in CDNs, the synchronization of CDNs has found a wide range of applications in a variety of research areas such as robotics system [25], communication engineering [32] and biological sciences [13], and a great many excellent results have been reported in the literature. So far, the most investigated synchronization mechanisms include phase synchronization, lag synchronization, complete synchronization, and cluster synchronization schemes, see, e.g., [3], [5], [6], [31], [36], [41], [47]-[51]. In particular, the cluster synchronization stands out as an interesting phenomenon with promising application potentials. For example, when a group of unmanned autonomous vehicles (connected by a local communication network) performs a complex task, the vehicles are divided into subgroups/clusters based on different subtasks, and vehicles within individual clusters are then required to achieve synchronization. Generally speaking, in cluster synchronization, the CDNs evolve into subgroups of nodes (called clusters) in which the nodes in the same cluster are synchronized with each other, but the nodes in different clusters might not.

In reality, it is quite common that an autonomous network is unable to achieve synchronization through its local connections or, in a larger scale, a CDN cannot achieve the desired self-synchronization status without exogenous interferences. In this case, it is natural to design certain control strategies, either open-loop or closed-loop, to help the underlying networks to accomplish the synchronization tasks. In fact, various control strategies have been designed in the literature for the synchronization purposes, see, for instance, feedback control [12], observer-based control [28], [44], pinning control [38], [43], adaptive control [35], and intermittent control protocols [21], [29].

It is noticeable that most existing synchronization control protocols have been developed for continuous-time systems/networks in the framework of analog communication. Nevertheless, with the burgeoning digital network technology, control systems have been undergoing continuous upgradation and the traditional analog communication is no longer sufficient to meet the communication needs of modern control systems. Instead, the digital communication strategies have proven to be particularly suitable for their distinct merits such as strong disturbance rejection capacity, high reliability, and low power consumption. In fact, digital communication mechanisms have been widely implemented in many areas including signal processing and control engineering [14], [20]. However, the synchronization control problem for CDNs subject to digital 
communication mechanism has not received much attention yet.

In engineering applications, almost all control or measurement signals are analog in nature. To be transmitted over a digital channel, such analog signals would need to go through the analog-to-digital conversion process which consists of sampling, quantization and coding steps. Generally speaking, sampling and quantization discretize the time and amplitude of the analog signal, respectively, and the coding process converts the quantized value into codewords composed of 0 and 1 following specific rules to adapt to digital communication. Despite its critically important role in digital communication, the coding process has attracted relatively little research attention as compared to the sampling/quantization counterparts [7], [15], [19], [39], [40]. It is worth pointing out that a typical design of coder-decoder pair in existing literature does not take into account the specific network bandwidth, which is actually an essential factor in designing the coder parameters.

For CDNs subject to digital communication mechanism, each node is usually allocated with only a small portion of the total bit rate according to the overall networks bandwidth available to the nodes. The bit rate, defined as the number of bits conveyed through a digital communication network per second, is the measure of network bandwidth in digital communication networks. Clearly, the bit rate constraints would have a major impact on analysis/synthesis of communicationbased systems/networks. To date, the stability analysis of feedback control loops under constrained bit rate has gain some initial attention [27], [37], [42], [45]. In existing results, the so-called data rate theorem has played an important role, which characterizes the minimum bit rate required to ensure various types of stability. For example, the minimum bit-rate conditions have been derived in [37] for asymptotic stability and in [45] for mean-square stabilizability of linear systems with Markovian packet losses [45].

In the past decade, an increasing research interest has been devoted to the investigation on the effect of constrained bit rate on the consensusability of multiagent systems [4], [17], [18], [46]. In the case of finite bit-rate communication, the average consensus control problem has been investigated in [17] for undirected networks with fixed and time-varying topologies of discrete-time agents. The joint effect of agent dynamics, network topology, and constrained bit rate on the consensusability of multiagent systems has been examined in [46]. For the quantized multiagent systems with the eventtriggering scheme, bit rate conditions for maintaining the desired asymptotic consensus have been derived in [4]. However, up to now, the cluster synchronization issue under constrained bit rate has not been adequately studied yet, which motivates us to fill in this gap in the current study.

In view of the above discussion, it is of both practical significance and theoretical importance to address the cluster synchronization control issue for CDNs subject to constrained bit rate. In this context, we foresee the following three substantial challenges: 1) how to develop a mathematical model that considers the total bandwidth limit of a CDN as well as the bandwidth allocation (of clusters and nodes)? 2) how to characterize the inherent relationship between the bit rate and the cluster synchronization control performance? and 3) how to design the controller gains according to some specific cluster synchronization performance requirements? To tackle the three identified challenges, this paper aims to investigate the cluster synchronization control problem for CDNs under constrained bit rate.

The main contributions of this paper are summarized in three aspects.

1) We have made a first attempt to deal with the problem of cluster synchronization in the framework of digital communication networks, which is more widely used in engineering application fields in comparison with the traditional cluster synchronization control strategies in the framework of analog communication networks [3], [21], [43]. And for the first time, a bit rate constraint model is introduced to characterize the network bandwidth of CDNs.

2) A sufficient condition pertaining to the bit rate is proposed to guarantee the ultimate boundedness of the cluster synchronization error dynamics, and a bit rate condition is subsequently proposed to guarantee the specific synchronization performance.

3) Two optimization problems (OPs) are proposed to obtain the desired gains of the cluster synchronization controllers to meet different synchronization performance metrics. Furthermore, the co-design problem with controller gains and bit rate allocation protocol as design parameters is considered for the first time.

Notation: In this paper, $\mathbb{N}$, and $\mathbb{N}^{+}$denote the sets of nonnegative integers, and positive integers, respectively. $\mathbb{R}^{n}$ and $\mathbb{R}^{n \times m}$ stand for $n$ dimensional Euclidean space and the set of $n \times m$ real matrices, respectively. $\operatorname{diag}_{N}\left\{A_{i}\right\}$ and $\operatorname{col}_{N}\left(e_{i}\right)$ denote diagonal block matrix $\operatorname{diag}_{N}\left\{A_{1}, A_{2}, \cdots, A_{N}\right\}$ and column vector $\left[e_{1}^{T}, e_{2}^{T}, \cdots, e_{N}^{T}\right]^{T}$, respectively. The notation $X>Y(X \geq Y)$ denotes that $X-Y$ is positive definite (semipositive definite), where $X$ and $Y$ are symmetric matrices. $\lambda_{\min }\{P\} \quad\left(\lambda_{\max }\{P\}\right)$ stands for the minimum (maximum) eigenvalue of $P$. For any $z \in \mathbb{R}^{n}, z^{T}$ and $\|z\|_{2}$ are its transpose and its Euclidean norm. For positive integers $k$ and $h, \bmod (k, h)$ denote the remainder on division of $k$ by $h$.

\section{Problem Formulation And Preliminaries}

\section{A. The system formulation}

A CDN with $N$ nonidentical nodes is described by the following form:

$$
\left\{\begin{aligned}
x_{i}(k+1) & =f_{i}\left(x_{i}(k)\right)+\sum_{j=1}^{N} \omega_{i j} \Gamma x_{j}(k)+u_{i}(k) \\
y_{i}(k) & =C_{i} x_{i}(k) \\
x_{i}(0) & =x_{i 0} \in \mathcal{I}_{0}, \quad i \in \mathcal{V} \triangleq\{1,2, \cdots, N\}
\end{aligned}\right.
$$

where $x_{i}(k) \in \mathbb{R}^{n_{x}}, y_{i}(k) \in \mathbb{R}^{n_{y}}$, and $u_{i}(k) \in \mathbb{R}^{n_{x}}$ represent the system state, the measurement output and the control input, respectively. $x_{i 0}$ is the initial value of the system belonging to a known set $\mathcal{I}_{0} . C_{i} \in \mathbb{R}^{n_{y} \times n_{x}}$ is a known matrix. The 
nonlinear function $f_{i}(\cdot)$ describes the local dynamics of node $i$ which satisfies $f_{i}(0)=0$ and the following condition:

$$
\begin{aligned}
\left(f_{i}\left(x_{1}\right)\right. & \left.-f_{i}\left(x_{2}\right)-\bar{u}_{i}\left(x_{1}-x_{2}\right)\right)^{T} \\
& \times\left(f_{i}\left(x_{1}\right)-f_{i}\left(x_{2}\right)-\underline{u}_{i}\left(x_{1}-x_{2}\right)\right) \leq 0
\end{aligned}
$$

for all $x_{1}, x_{2} \in \mathbb{R}^{n_{x}}$, where $\bar{u}_{i}$ and $\underline{u}_{i}$ are known real-valued matrices.

Let the CDN (1) be divided into $m$ clusters with $\mathcal{V}_{1}=$ $\left\{1,2, \cdots, n_{1}\right\}, \mathcal{V}_{2}=\left\{n_{1}+1, \cdots, n_{1}+n_{2}\right\}, \cdots, \mathcal{V}_{m}=$ $\left\{\sum_{l=1}^{m-1} n_{l}+1, \cdots, \sum_{l=1}^{m} n_{l}\right\}$ where, for $l \in \Phi^{m} \triangleq$ $\{1,2, \cdots, m\}, 1 \leq n_{l}<N$ and $\sum_{l=1}^{m} n_{l}=N$. In other words, we have $\mathcal{V}_{l} \neq \emptyset$ and $\bigcup_{l=1}^{m} \mathcal{V}_{l}=\mathcal{V}$. Furthermore, the symbol $\bar{i}$ is used to indicate which cluster the $i$ th node belongs to, i.e., $i \in \mathcal{V}_{\bar{i}}$. Obviously, for nodes $i$ and $j$ in the same cluster, $\bar{i}=\bar{j}$. Next, we make the following assumption that nodes in the same cluster have the same local dynamics, i.e., for $\bar{i}=\bar{j}$, $f_{\bar{i}}(\cdot)=f_{\bar{j}}(\cdot)$.

The coupled configuration matrix $\mathcal{W}=\left[\omega_{i j}\right]_{N \times N}$, which denotes the topology of the CDN with $m$ clusters, is assumed to be symmetric and irreducible with the following form:

$$
\mathcal{W}=\left[\begin{array}{cccc}
\mathcal{W}_{11} & \mathcal{W}_{12} & \cdots & \mathcal{W}_{1 m} \\
\mathcal{W}_{21} & \mathcal{W}_{22} & \cdots & \mathcal{W}_{2 m} \\
\vdots & \vdots & \ddots & \vdots \\
\mathcal{W}_{d 1} & \mathcal{W}_{d 2} & \cdots & \mathcal{W}_{m m}
\end{array}\right]
$$

where each matrix block $\mathcal{W}_{s t}=\left[\omega_{i j}\right]_{n_{s} \times n_{t}} \in \mathbb{R}^{n_{s} \times n_{t}}$ is a zero-row-sum matrix, i.e., $\sum_{j=1}^{n_{t}} \omega_{i j}=0$, and each diagonal matrix block $\mathcal{W}_{s s}=\left[\omega_{i j}\right]_{n_{s} \times n_{s}} \in \mathbb{R}^{n_{s} \times n_{s}}$ satisfies $\omega_{i i}=$ $-\sum_{j=1, j \neq i}^{N} \omega_{i j}$. Moreover, $\omega_{i j}=\omega_{j i}>0$ if there exists a connection between the node $i$ and node $j$. In particular, the inter-cluster couplings $\omega_{i j}$ with $i \in \mathcal{V}_{s}$ and $j \in \mathcal{V}_{t}$ can be negative here, which provides a mechanism to desynchronize two nodes belonging to two different clusters. The innercoupling matrix $\Gamma=\operatorname{diag}\left\{\gamma_{1}, \gamma_{2}, \cdots, \gamma_{n_{x}}\right\}$ represents the connections between the different elements of the subsystem, where $\gamma_{l} \neq 0$ means that the $l$ th component of $x_{j}(k)$ has an impact on the $x_{i}(k)$.

It should be noted that, when designing the cluster synchronization controller, it may be possible that the complete state information of the CDN (1) is not fully accessible, but only the measured output of the system is available. As such, the following state estimator is constructed to estimate the system state based on the measured output:

$$
\left\{\begin{aligned}
\hat{x}_{i}(k+1)= & f_{i}\left(\hat{x}_{i}(k)\right)+\sum_{j=1}^{N} \omega_{i j} \Gamma \hat{x}_{j}(k) \\
& +L_{i}\left(y_{i}(k)-C_{i} \hat{x}_{i}(k)\right)+u_{i}(k) \\
\hat{x}_{i}(0)= & \hat{x}_{i 0} \in \mathcal{I}_{0}
\end{aligned}\right.
$$

where $\hat{x}_{i}(k) \in \mathbb{R}^{n_{x}}$ and $\hat{x}_{i}(0)$ are the state estimate and the initial condition, respectively, and $L_{i} \in \mathbb{R}^{n_{x} \times n_{y}}$ is the estimator gain to be designed.

For the $l$ th cluster, the target trajectory is denoted as $t_{l}(k) \in$ $\mathbb{R}^{n_{x}}$, which is the solution to the following dynamics of the unforced isolated node:

$$
\left\{\begin{aligned}
t_{l}(k+1) & =f_{l}\left(t_{l}(k)\right) \\
t_{l}(0) & =t_{l 0} \in \mathcal{I}_{0}
\end{aligned}\right.
$$

where $t_{l 0}$ is the initial condition, and $f_{l}\left(t_{l}(k)\right)$ characterizes the local dynamics of the nodes in the $l$ th cluster. Therefore, for $i \in \mathcal{V}_{l}, f_{i}\left(x_{i}(k)\right)=f_{l}\left(t_{l}(k)\right)$. In particular, for $l, j \in \Phi^{m}$ and $l \neq j, f_{l}\left(t_{l}(k)\right) \neq f_{j}\left(t_{j}(k)\right)$.

\section{B. Description of the bit rate}

In this paper, the transmission of coded information is implemented by applying a wireless digital communication network under bit rate constraint.

In real CDNs, the available bandwidth of a network is usually limited due to the hardware and network communication capabilities. So far, a variety of channel allocation protocols have been applied to allocate specific bit rates to different clusters and nodes in order to reduce data collisions. A typical model of bit rate constraint is expressed as follows:

$$
\begin{gathered}
\sum_{l=1}^{m} R_{l} \leq R_{s} \\
\sum_{i \in \mathcal{V}_{l}} R_{l, i} \leq R_{l}
\end{gathered}
$$

where $R_{s} \in \mathbb{N}^{+}$represents the total available bit rate determined by the physical devices, $R_{l} \in \mathbb{N}$ denotes the allocated bit rate of cluster $l$, and $R_{l, i}$ indicates the allocated bit rate of node $i$ in cluster $l$. To facilitate the presentation, we denote the allocated bit rate of node $i$ by $R_{\bar{i} i}$.

Remark 1: In order to reduce data collisions under limited bandwidth, the allocation-based media access control (MAC) protocols are widely used in large-scale networks such as CDNs and sensor networks [1], [30]. According to the cluster network characteristics, the allocation-based MAC protocols are used to allocate the available bit rate of each cluster (and each node in the cluster) through the relevant protocol. Therefore, as presented in (6) and (7), a bit rate model would include two constraints, which represent the bandwidth allocation rules for the clusters within the $\mathrm{CDN}$ and for the nodes within a cluster.

\section{Cluster synchronization under coding-decoding procedure}

In this paper, the transmission of information occurs at $d h$ moments, and the transmitted information is a string of binary codes selected from an alphabet $\mathbb{A}_{R_{\bar{i} i}}$ of size $2^{R_{\bar{i} i}}$, where $h$ is a given coding interval, $d=1,2, \cdots$, and $\mathbb{A}_{R_{\bar{i} i}}$ is the alphabet in the terms of bit rate $R_{\bar{i} i}$. Obviously, the bit rate has an important impact on the alphabet's size, and thus the bit rate constraints (6) and (7) will be taken into account in the subsequent design of the coding-decoding strategy. A general form of the coding-decoding procedure is given as follows.

Coder of node $i$ under bit rate $R_{\bar{i} i}$.

$$
\mathcal{X}_{i}^{R_{\bar{i} i}}(d h)=\mathcal{C}_{i}^{R_{\bar{i} i}, d}\left(\hat{x}_{i}(h), \hat{x}_{i}(2 h) \cdots, \hat{x}_{i}(d h)\right)
$$

\section{Decoder of node $i$.}

$$
\breve{\mathcal{X}}_{i}(d h)=\mathcal{D}_{i}^{d}\left(\mathcal{X}_{i}^{R_{\bar{i} i}}(h), \mathcal{X}_{i}^{R_{\bar{i} i}}(2 h), \cdots, \mathcal{X}_{i}^{R_{\bar{i} i}}(d h)\right)
$$


for $d=1,2, \cdots$, where $\mathcal{C}_{i}^{R_{\bar{i}}, d}(\cdot)$ and $\mathcal{D}_{i}^{d}(\cdot)$ are the coder and decoder functions, $\mathcal{X}_{i}^{R_{\bar{i} i}}(d h)$ is the codeword generated by node $i$ at the coding constant $d h$, and $\breve{\mathcal{X}}_{i}(d h)$ is denoted as

$$
\breve{\mathcal{X}}_{i}(d h)=\left\{\breve{x}_{i}(d h), \breve{x}_{i}(d h+1) \cdots, \breve{x}_{i}((d+1) h-1)\right\}
$$

with $\breve{x}_{i}(k)$ being the decoded output of $\hat{x}_{i}(k)$ for $k \in[d h,(d+$ 1)h).

Due to the coding-decoding procedure described previously, the network-based controller obtains $\breve{x}_{i}(k)$ (instead of the original estimate $\left.\hat{x}_{i}(k)\right)$. In this case, the decoder-based controller for node $i$ is designed as

$$
u_{i}(k)=K_{i}\left(\breve{x}_{i}(k)-t_{\bar{i}}(k)\right) .
$$

Note that, when it comes to the estimator design, it is impractical (also impossible) to feed the decoded signal $\breve{x}_{i}(k)$ or the control signal $u_{i}(k)$ back to the state estimator due to the network bandwidth limitation. To overcome this problem, the auxiliary control signal $\check{u}_{i}(k)=K_{i}\left(\check{x}_{i}(k)-t_{\bar{i}}(k)\right)$ defined in III-A [see (23)] is used to design the following state estimator:

$$
\left\{\begin{aligned}
\hat{x}_{i}(k+1)= & f_{i}\left(\hat{x}_{i}(k)\right)+\sum_{j=1}^{N} \omega_{i j} \Gamma \hat{x}_{j}(k) \\
& +L_{i}\left(y(k)-C_{i} \hat{x}_{i}(k)\right)+\check{u}_{i}(k) \\
\hat{x}_{i}(0)= & \hat{x}_{i 0} \in \mathcal{I}_{0}
\end{aligned}\right.
$$

where $\check{u}_{i}(k)$ is the same as $u_{i}(k)$ according to (23) and (24) defined in III-A.

The synchronization error of the $i$ th node is defined as $e_{i}(k)=x_{i}(k)-t_{\bar{i}}(k)$. By considering the properties of the coupling matrix, we obtain

$$
\begin{aligned}
\sum_{j=1}^{N} \omega_{i j} \Gamma x_{j}(k) & =\sum_{l=1}^{m} \sum_{j \in \mathcal{V}_{l}} \omega_{i j} \Gamma x_{j}(k) \\
& =\sum_{l=1}^{m} \sum_{j \in \mathcal{V}_{l}} \omega_{i j} \Gamma\left[x_{j}(k)-t_{l}(k)+t_{l}(k)\right] \\
& =\sum_{l=1}^{m} \sum_{j \in \mathcal{V}_{l}} \omega_{i j} \Gamma\left[e_{j}(k)+t_{l}(k)\right] \\
& =\sum_{l=1}^{m} \sum_{j \in \mathcal{V}_{l}} \omega_{i j} \Gamma e_{j}(k)+\sum_{l=1}^{m} \sum_{j \in \mathcal{V}_{l}} \omega_{i j} \Gamma t_{l}(k) \\
& =\sum_{j=1}^{N} \omega_{i j} \Gamma e_{j}(k) .
\end{aligned}
$$

Then, the corresponding cluster synchronization error dynamics of node $i$ is obtained as:

$$
\left\{\begin{aligned}
e_{i}(k+1) & =f_{i}\left(e_{i}(k)\right)+\sum_{j=1}^{N} \omega_{i j} \Gamma e_{j}(k)+u_{i}(k) \\
e_{i}(0) & =e_{i 0} \in \mathcal{I}_{0}
\end{aligned}\right.
$$

where $f_{i}\left(e_{i}(k)\right)=f_{i}\left(x_{i}(k)\right)-f_{i}\left(t_{\bar{i}}(k)\right)$, and $e_{i 0}=x_{i 0}-t_{\bar{i} 0}$ is the initial value of the cluster synchronization error.

To simplify the symbolic representation, we set

$$
x(k)=\operatorname{col}_{N}\left(x_{i}(k)\right), y(k)=\operatorname{col}_{N}\left(y_{i}(k)\right)
$$

$$
\begin{aligned}
\breve{x}(k) & =\operatorname{col}_{N}\left(\breve{x}_{i}(k)\right), \quad e(k)=\operatorname{col}_{N}\left(e_{i}(k)\right) \\
\hat{x}(k) & =\operatorname{col}_{N}\left(\hat{x}_{i}(k)\right), \quad \check{x}(k)=\operatorname{col}_{N}\left(\check{x}_{i}(k)\right) \\
\hat{x}_{0} & =\operatorname{col}_{N}\left(\hat{x}_{i 0}\right), x_{0}=\operatorname{col}_{N}\left(x_{i 0}\right), e_{0}=\operatorname{col}_{N}\left(e_{i 0}\right) \\
F(x(k)) & =\operatorname{col}_{N}\left(f_{i}\left(x_{i}(k)\right)\right), F(e(k))=\operatorname{col}_{N}\left(f_{i}\left(e_{i}(k)\right)\right) \\
C & =\operatorname{diag}_{N}\left\{C_{i}\right\}, L=\operatorname{diag}_{N}\left\{L_{i}\right\} \\
K & =\operatorname{diag}_{N}\left\{K_{i}\right\}, \hat{t}(k)=\operatorname{col}_{N}\left(t_{\bar{i}}(k)\right) .
\end{aligned}
$$

By resorting to the Kronecker product, the CDN (1) is rearranged as follows:

$$
\left\{\begin{aligned}
x(k+1) & =F(x(k))+(\mathcal{W} \otimes \Gamma) x(k)+K(\breve{x}(k)-\hat{t}(k)) \\
y(k) & =C x(k) \\
x(0) & =x_{0}
\end{aligned}\right.
$$

and the corresponding estimator of the augmented CDN (14) is reformulated as follows:

$$
\left\{\begin{aligned}
\hat{x}(k+1)= & F(\hat{x}(k))+(\mathcal{W} \otimes \Gamma) \hat{x}(k) \\
& +L C(x(k)-\hat{x}(k))+K(\check{x}(k)-\hat{t}(k)) \\
\hat{x}(0)= & \hat{x}_{0} .
\end{aligned}\right.
$$

Correspondingly, the cluster synchronization error dynamics can be rearranged as the following form:

$$
\left\{\begin{aligned}
e(k+1) & =F(e(k))+(\mathcal{W} \otimes \Gamma) e(k)+K(\breve{x}(k)-\hat{t}(k)) \\
e(0) & =e_{0} .
\end{aligned}\right.
$$

We are now ready to state the main problem addressed in this paper. We are interested in dealing with the cluster synchronization control problem with constrained bit rate whose schematic structure is depicted in Fig. 1. The main objective is to design controllers for a CDN with $N$ coupled nonidentical nodes (which can be divided into $m$ clusters) such that the dynamics of the cluster synchronization error system is ultimately bounded subject to the decoding error $d_{e}(k)$.

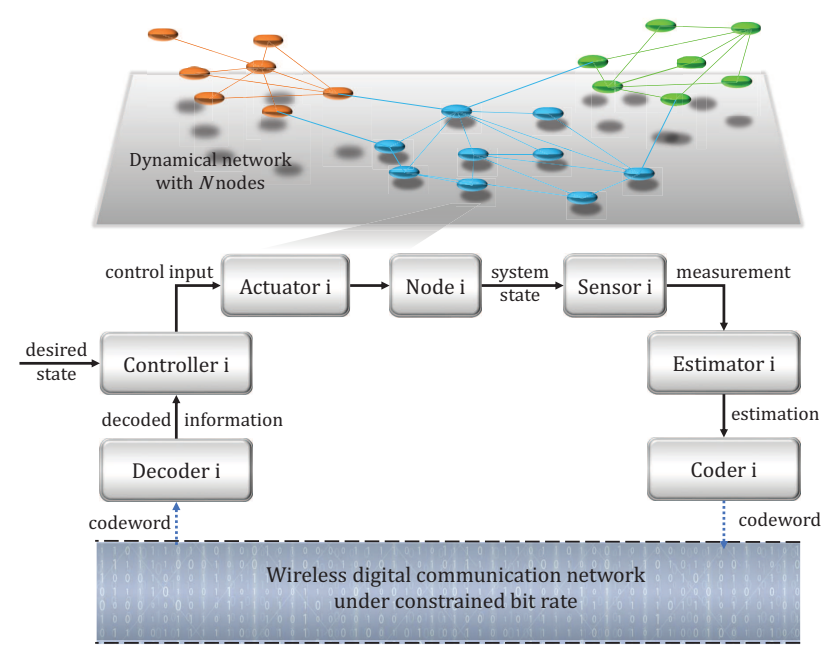

Fig. 1. Cluster synchronization problem over limited digital communication network

Before proceeding further, the following definitions are presented to assist in the derivation of our main results. 
Definition 1: [55] The dynamics of the cluster synchronization error $e(k)$ [i.e., the solution of system (16)] is said to be exponentially ultimately bounded if there exist constants $\sigma>0, \rho>0$, and $\phi>0$, such that

$$
\|e(k)\|_{2}^{2} \leq \sigma^{k} \rho+\phi
$$

where $\sigma \in[0,1)$ is the decay rate and $\phi$ is the asymptotic upper bound (AUB) of $\|e(k)\|_{2}^{2}$.

Definition 2: Under the bit rate constraints (6) and (7), the discrete-time CDN (1) with $N$ nonidentical nodes is said to achieve exponentially ultimately bounded cluster synchronization if the $N$ nodes are split into $m$ clusters $\mathcal{V}_{1}, \mathcal{V}_{2}, \cdots, \mathcal{V}_{m}$, and the cluster synchronization error dynamics (16) is exponentially ultimately bounded.

\section{Main Results}

In this section, we plan to first discuss the design problem of the coding-decoding procedure for CDN (1), and then based on the established coding-decoding procedure, we address the analysis and design problem of cluster synchronization control under the bit rate constraints (6) and (7).

\section{A. Coding-decoding procedure under constrained bit rate}

In this subsection, we are going to take bit rate constraints (6) and (7) into account for the design of the coding-decoding process.

1. The coding procedure of node $i$ under bit rate $R_{\bar{i} i}$.

The following uniform quantizer is adopted in this paper to improve the coding-decoding procedure. For node $i$, given a scaling parameter $b_{i}>0$, the quantization region is determined subsequently as

$$
\mathcal{B}_{b_{i}} \triangleq\left\{e_{x i} \in \mathbb{R}^{n_{x}}:\left|e_{x i}^{(j)}\right| \leq b_{i}, j=1,2, \cdots, n_{x}\right\},
$$

where $e_{x i}^{(j)}$ is the $j$ th element of the vector $e_{x i}$.

By choosing an integer $q_{i}$ as the number of quantization levels, the hyperrectangles $\mathcal{B}_{b_{i}}$ will be partitioned into $q_{i}^{n_{x}}$ sub-hyperrectangles $I_{s_{1}^{i}}^{i 1}\left(b_{i}\right) \times I_{s_{2}^{i}}^{i 2}\left(b_{i}\right) \times \cdots \times I_{s_{n_{x}}^{i}}^{i i_{x}}\left(b_{i}\right)$, with $s_{1}^{i}, s_{2}^{i}, \cdots, s_{n_{x}}^{i} \in\left\{1,2, \cdots, q_{i}\right\}$ and

$$
\begin{aligned}
I_{1}^{i j}\left(b_{i}\right) \triangleq & \left\{e_{x i}^{(j)} \mid-b_{i} \leq e_{x i}^{(j)}<-b_{i}+\frac{2 b_{i}}{q_{i}}\right\} \\
I_{2}^{i j}\left(b_{i}\right) \triangleq & \left\{e_{x i}^{(j)} \mid-b_{i}+\frac{2 b_{i}}{q_{i}} \leq e_{x i}^{(j)}<-b_{i}+\frac{4 b_{i}}{q_{i}}\right\} \\
& \vdots \\
I_{q_{i}}^{i j}\left(b_{i}\right) \triangleq & \left\{e_{x i}^{(j)} \mid b_{i}-\frac{2 b_{i}}{q_{i}} \leq e_{x i}^{(j)} \leq b_{i}\right\} .
\end{aligned}
$$

To ensure that the information corresponding to each subhyperrectangle is encoded uniquely, the number of quantization levels needs to be designed according to the network's bit rate. Thus, when the available bit rate of node $i$ is allocated as $R_{\bar{i} i}$ subject to the bit rate constraints (6) and (7), the maximum number of quantization levels is defined as

$$
q_{i m}=\left\lfloor\sqrt[n_{x}]{2^{R_{\bar{i}}}}\right\rfloor
$$

where $\left[\sqrt[n_{x}]{2^{R_{\bar{i} i}}}\right\rfloor$ describes the maximum integer less than or equal to $\sqrt[n_{x}]{2^{R_{\bar{i}}}}$.

For each $\mathcal{B}_{b_{i}}$, the center of the hyperrectangle $I_{s_{1}^{i}}^{i 1}\left(b_{i}\right) \times$ $I_{s_{2}^{i}}^{i 2}\left(b_{i}\right) \times \cdots \times I_{s_{n_{x}}^{i}}^{i n_{x}}\left(b_{i}\right)$ is denoted by

$$
\hbar_{b_{i}}^{i}\left(s_{1}^{i}, s_{2}^{i}, \cdots, s_{n_{x}}^{i}\right) \triangleq\left[\begin{array}{llll}
c_{i 1} & c_{i 2} & \cdots & c_{i n_{x}}
\end{array}\right]^{T}
$$

with

$$
c_{i j}=-b_{i}+\left[\left(\left(2 s_{j}^{i}-1\right) b_{i}\right) /\left[\sqrt[n_{x}]{2^{R_{\bar{i} i}}}\right\rfloor\right]
$$

for $j=1,2, \cdots, n_{x}$. Hence, for any $e_{x i} \in \mathcal{B}_{b_{i}}$, there exists a certain set of integers $s_{1}^{i}, s_{2}^{i}, \cdots, s_{n_{x}}^{i} \in\left\{1,2, \cdots, q_{i}\right\}$ such that $e_{x i} \in I_{s_{1}^{i}}^{i 1} \times I_{s_{2}^{i}}^{i 2} \times \cdots \times I_{s_{n_{x}}^{i}}^{i n_{x}}$, which satisfies the following inequality:

$$
\left\|e_{x i}-\hbar_{b_{i}}^{i}\left(s_{1}^{i}, s_{2}^{i}, \cdots, s_{n_{x}}^{i}\right)\right\|_{2} \leq \frac{\sqrt{n_{x}} b_{i}}{\left[\sqrt[n_{x}]{2^{R_{\bar{i} i}}}\right]} .
$$

Here, the integers $s_{1}^{i}, s_{2}^{i}, \cdots, s_{n_{x}}^{i} \in\left\{1,2, \cdots, q_{i m}\right\}$ are the components of the codeword in the coding procedure.

With the preparation made so far, the coder is now designed as follows.

For $e_{x i}(d h)=\hat{x}_{i}(d h)-\bar{x}_{i}(d h) \in I_{s_{1}^{i}}^{i 1}\left(b_{i}\right) \times I_{s_{2}^{i}}^{i 2}\left(b_{i}\right) \times \cdots \times$ $I_{s_{n_{n}}^{i}}^{i n_{x}}\left(b_{i}\right) \subset \mathcal{B}_{b_{i}}, d=1,2, \cdots$, the following codeword is generated

$$
\mathcal{X}_{i}^{R_{\bar{i} i}}(k)=\left[s_{1}^{i}, \cdots, s_{n_{x}}^{i}\right]
$$

where $\hat{x}_{i}(d h)$ is the state estimate of node $i$ at coding instant $d h, \bar{x}_{i}(d h)$ is determined by

$$
\left\{\begin{aligned}
\bar{x}_{i}(0)= & \\
\bar{x}_{i}(k)= & \check{x}_{i}(k), k \neq d h \\
\bar{x}_{i}(d h)= & f_{i}\left(\check{x}_{i}(d h-1)\right)+\sum_{j=1}^{N} \omega_{i j} \Gamma \check{x}_{j}(d h-1) \\
& +\check{u}_{i}(d h-1) \\
\check{u}_{i}(d h-1)= & K_{i}\left(\check{x}_{i}(d h-1)-t_{\bar{i}}(d h-1)\right)
\end{aligned}\right.
$$

and the dynamics of $\breve{x}_{i}(k)$ is governed by

$$
\left\{\begin{aligned}
\check{x}_{i}(0) & =0 \\
\check{x}_{i}(k+1) & =f_{i}\left(\check{x}_{i}(k)\right)+\sum_{j=1}^{N} \omega_{i j} \Gamma \check{x}_{j}(k)+\check{u}_{i}(k), \\
\check{x}_{i}(d h) & =\bar{x}_{i}(d h)+\hbar_{b_{i}}^{i}\left(s_{1}^{i}, s_{2}^{i}, \cdots, s_{n_{x}}^{i}\right) \\
\check{u}_{i}(k) & =K_{i}\left(\check{x}_{i}(k)-t_{\bar{i}}(k)\right)
\end{aligned}\right.
$$

where $\breve{x}_{i}(k)$ denote the state of the auxiliary system (23).

2. Decoding procedure of node $i$.

The decoder is designed as follows:

$$
\left\{\begin{aligned}
\breve{x}_{i}(0) & =0 \\
\breve{x}_{i}(k+1) & =f_{i}\left(\breve{x}_{i}(k)\right)+\sum_{j=1}^{N} \omega_{i j} \Gamma \breve{x}_{j}(k)+u_{i}(k), \\
\quad \text { for } k \neq d h-1 & \\
\breve{x}_{i}(d h) & =\bar{x}_{i}(d h)+\hbar_{b_{i}}^{i}\left(s_{1}^{i}, s_{2}^{i}, \cdots, s_{n_{x}}^{i}\right) \\
u_{i}(k) & =K_{i}\left(\breve{x}_{i}(k)-t_{\bar{i}}(k)\right)
\end{aligned}\right.
$$


where $\breve{x}_{i}(k)$ is the state of decoded output.

Remark 2: In this paper, $\hat{x}_{i}(d h)-\bar{x}_{i}(d h)$ is coded at each coding instant $d h$ rather than $\hat{x}_{i}(d h) . \bar{x}_{i}(d h)$ is designed to predict the decoder state $\breve{x}_{i}(k)$, which overcomes the practical difficulty that the coder is incapable of obtaining the decoder state. The auxiliary system (23) is designed as $\breve{x}_{i}(k) \equiv \breve{x}_{i}(k)$ for $\forall k \geq 0$. This design method is reasonable as it removes the unrealistic requirement of returning the decoded state to the coder.

Denoting

$$
\begin{aligned}
& e_{x}(k)=\operatorname{col}_{N}\left(e_{x i}(k)\right), \quad F(\hat{x}(k))=\operatorname{col}_{N}\left(f_{i}\left(\hat{x}_{i}(k)\right)\right), \\
& F(\check{x}(k))=\operatorname{col}_{N}\left(f_{i}\left(\check{x}_{i}(k)\right)\right), \quad F(\breve{x}(k))=\operatorname{col}_{N}\left(f_{i}\left(\breve{x}_{i}(k)\right)\right),
\end{aligned}
$$

the coder-decoder pair of the CDN is reformulated in the following compact form.

Coder under constrained bit rate $R_{s}$ : For $e_{x}(d h)=$ $\hat{x}(d h)-\bar{x}(d h) \in I_{s_{1}^{1}}^{11}\left(b_{1}\right) \times \cdots \times I_{s_{n_{x}}^{1}}^{1 n_{x}}\left(b_{1}\right) \times I_{s_{1}^{2}}^{21}\left(b_{2}\right) \times \cdots \times$ $I_{s_{n_{x}}^{2}}^{2 n_{x}}\left(b_{2}\right) \times \cdots \times I_{s_{1}^{N}}^{N 1}\left(b_{N}\right) \times \cdots \times I_{s_{n_{x}}^{N}}^{N n_{x}}\left(b_{N}\right) \subset \mathcal{B}_{\bar{b}}, d=1,2, \cdots$, the following codeword is generated:

$$
\mathcal{X}^{R_{s}}(k)=\left[s_{1}^{1}, \cdots, s_{n_{x}}^{1}, s_{1}^{2}, \cdots, s_{n_{x}}^{2}, \cdots, s_{1}^{N}, \cdots, s_{n_{x}}^{N}\right]
$$

where $\mathcal{B}_{b}=\left\{e_{x}(d h) \in \mathbb{R}^{N n_{y}}:\left|e_{x}^{(j)}(d h)\right| \leq \bar{b}, j=\right.$ $\left.1,2, \cdots, N n_{x}\right\}$ with $\bar{b}=\max _{1 \leq i \leq N}\left\{b_{i}\right\}$ and $\bar{x}(d h)=$ $\operatorname{col}_{N}\left(\bar{x}_{i}(d h)\right)$ is determined by

$$
\left\{\begin{aligned}
\bar{x}(0)= & 0 \\
\bar{x}(k)= & \check{x}(k), \quad k \neq d h \\
\bar{x}(d h)= & F(\check{x}(d h-1))+(\mathcal{W} \otimes \Gamma) \check{x}(d h-1) \\
& +\check{u}(d h-1) \\
\check{u}(d h-1)= & K(\check{x}(d h-1)-t(d h-1))
\end{aligned}\right.
$$

and the dynamics of $\check{x}_{i}(k)$ evolves as follows:

$$
\left\{\begin{aligned}
\check{x}(0) & =0 \\
\check{x}(k+1) & =F(\check{x}(k))+(\mathcal{W} \otimes \Gamma) \check{x}(k)+\check{u}(k), k \neq d h-1 \\
\check{x}(d h) & =\bar{x}(d h)+\hbar_{b}\left(s_{1}^{1}, \cdots, s_{n_{x}}^{N}\right) \\
\check{u}(k) & =K(\check{x}(k)-t(k)) .
\end{aligned}\right.
$$

Decoder: The compact form of decoder is given as follow:

$$
\left\{\begin{aligned}
\breve{x}(0) & =0 \\
\breve{x}(k+1) & =F(\breve{x}(k))+(\mathcal{W} \otimes \Gamma) \breve{x}(k)+u(k), k \neq d h-1 \\
\breve{x}(d h) & =\bar{x}(d h)+\hbar_{b}\left(s_{1}^{1}, \cdots, s_{n_{x}}^{N}\right) \\
u(k) & =K(\breve{x}(k)-t(k))
\end{aligned}\right.
$$

where

$$
\hbar_{b}\left(s_{1}^{1}, \cdots, s_{n_{x}}^{N}\right)=\operatorname{col}_{N}\left(\hbar_{b_{i}}^{i}\left(s_{1}^{i}, \cdots, s_{n_{x}}^{i}\right)\right) .
$$

Furthermore, one derives from (20) that

$$
\left\|e_{x}-\hbar_{b}\left(s_{1}^{1}, \cdots, s_{n_{x}}^{N}\right)\right\|_{2} \leq \sqrt{\sum_{i=1}^{N} \frac{n_{x} b_{i}^{2}}{\left[\sqrt[n_{x}]{2^{R_{\bar{i}}}}\right]^{2}}} .
$$

To facilitate the analysis of the cluster synchronization control afterwards, we give the following two lemmas.
Lemma 1: Let the scalar $\eta_{1}>0$ be given. If there exists a positive definite matrix $Q_{1}$ and scalar $\varepsilon_{1}$ satisfying

$$
\Pi_{1}=\left[\begin{array}{ccc}
\Pi_{11} & \varepsilon_{1} \hat{U}_{2} & (\mathcal{W} \otimes \Gamma)^{T} Q_{1} \\
* & -\varepsilon_{1} I & Q_{1} \\
* & * & -Q_{1}
\end{array}\right]<0
$$

with $\Pi_{11}=-\left(1+\eta_{1}\right) Q_{1}-\varepsilon_{1} \hat{U}_{1}$, then, the following inequality

$$
\left\|\varpi_{1}(k+1)-\varpi_{2}(k+1)\right\|_{2} \leq p_{1}\left\|\varpi_{1}(k)-\varpi_{2}(k)\right\|_{2}
$$

is satisfied, where $p_{1}=\sqrt{\rho_{1}}, \rho_{1} \triangleq\left(1+\eta_{1}\right) \bar{\lambda}_{Q_{1}} \underline{\lambda}_{Q_{1}}^{-1}$, $\bar{\lambda}_{Q_{1}} \triangleq \lambda_{\max }\left\{Q_{1}\right\}, \underline{\lambda}_{Q_{1}} \triangleq \lambda_{\min }\left\{Q_{1}\right\} ; \varpi_{1}(k)$ and $\varpi_{2}(k)$ are two solutions of (14).

Proof: Letting $v(k) \triangleq \varpi_{1}(k)-\varpi_{2}(k)$ and $F(v(k)) \triangleq$ $F\left(\varpi_{1}(k)\right)-F\left(\varpi_{2}(k)\right)$, it can be deduced from (14) that $v(k+1)=F(v(k))+(\mathcal{W} \otimes \Gamma) v(k)$. By constructing a Lyapunov function $V_{1}(k)=v^{T}(k) Q_{1} v(k)$, the difference of $V_{1}(k)$ is defined as $\Delta V_{1}(k) \triangleq V_{1}(k+1)-V_{1}(k)$. Then, the term $\Delta V_{1}(k)-\eta_{1} V_{1}(k)$ can be calculated as follows:

$$
\begin{aligned}
& \Delta V_{1}(k)-\eta_{1} V_{1}(k) \\
= & v^{T}(k+1) Q_{1} v(k+1)-\left(1+\eta_{1}\right) v^{T}(k) Q_{1} v(k) \\
= & v^{T}(k)\left[(\mathcal{W} \otimes \Gamma)^{T} Q_{1}(\mathcal{W} \otimes \Gamma)-\left(1+\eta_{1}\right) Q_{1}\right] v(k) \\
& +F^{T}(v(k)) Q_{1} F(v(k))+2 F^{T}(v(k)) Q_{1}(\mathcal{W} \otimes \Gamma) v(k) .
\end{aligned}
$$

It follows from (2) that

$$
\varepsilon_{1}\left[\begin{array}{c}
v(k) \\
F(v(k))
\end{array}\right]^{T}\left[\begin{array}{cc}
\hat{U}_{1} & -\hat{U}_{2} \\
* & I
\end{array}\right]\left[\begin{array}{c}
v(k) \\
F(v(k))
\end{array}\right] \leq 0
$$

where $\hat{U}_{1}=\operatorname{diag}_{N}\left\{\bar{U}_{i}\right\}, \hat{U}_{2}=\operatorname{diag}_{N}\left\{\tilde{U}_{i}\right\}, \bar{U}_{i}=\left(\bar{u}_{i}^{T} \underline{u}_{i}+\right.$ $\left.\bar{u}_{i} \underline{u}_{i}^{T}\right) / 2$, and $\tilde{U}_{i}=\left(\bar{u}_{i}+\underline{u}_{i}\right) / 2$. Then, for any positive scalar $\varepsilon_{1}$, one has

$$
\Delta V_{1}(k)-\eta_{1} V_{1}(k) \leq \xi^{T}(k) \bar{\Pi}_{1} \xi(k)
$$

where

$$
\begin{aligned}
\bar{\Pi}_{1} & =\left[\begin{array}{cc}
\bar{\Pi}_{11} & (\mathcal{W} \otimes \Gamma)^{T} Q_{1}+\varepsilon_{1} \hat{U}_{2} \\
* & Q_{1}-\varepsilon_{1} I
\end{array}\right] \\
\bar{\Pi}_{11} & =(\mathcal{W} \otimes \Gamma)^{T} Q_{1}(\mathcal{W} \otimes \Gamma)-\left(1+\eta_{1}\right) Q_{1}-\varepsilon_{1} \hat{U}_{1} \\
\xi(k) & =\left[\begin{array}{ll}
v^{T}(k) & F^{T}(v(k))
\end{array}\right]^{T} .
\end{aligned}
$$

Applying Schur Complement Lemma to (31), it is readily seen that $\bar{\Pi}_{1}<0$. Recalling the definition of $V_{1}(k)$, we arrive at $\lambda_{\min }\left\{Q_{1}\right\}\|v(k+1)\|_{2}^{2} \leq V_{1}(k+1) \leq(1+$ $\left.\eta_{1}\right) \lambda_{\max }\left\{Q_{1}\right\}\|v(k)\|_{2}^{2}$. It is easily seen that $\|v(k+1)\|_{2} \leq$ $\sqrt{\rho_{1}}\|v(k)\|_{2}$, which indicates $\left\|\varpi_{1}(k+1)-\varpi_{2}(k+1)\right\|_{2} \leq$ $p_{1}\left\|\left(\varpi_{1}(k)-\varpi_{2}(k)\right)\right\|_{2}$. The proof is complete.

Based on the results of Lemma 1, a sufficient condition to guarantee the convergence of the estimation error dynamics for the state estimator design problem is given in the following Lemma.

Lemma 2: Let the positive scalar $0<\eta_{2}<1$ be given. For the CDN (14) with state estimator (15), assume that there exist 
a positive definite matrix $\mathcal{Q}_{2}=\operatorname{diag}\left\{Q_{2}, Q_{3}, \cdots, Q_{N+1}\right\}$, a matrix $\bar{L}=\operatorname{diag}_{N}\left\{\bar{L}_{i}\right\}$, and a positive scalar $\varepsilon_{2}$ satisfying

$$
\Pi_{2}=\left[\begin{array}{ccc}
\Pi_{21} & \varepsilon_{2} \hat{U}_{2} & \Pi_{22} \\
* & -\varepsilon_{2} I & \mathcal{Q}_{2} \\
* & * & -\mathcal{Q}_{2}
\end{array}\right]<0
$$

with $\Pi_{21}=-\left(1-\eta_{2}\right) \mathcal{Q}_{2}-\varepsilon_{2} \hat{U}_{1}$ and $\Pi_{22}=\left(\mathcal{Q}_{2}(\mathcal{W} \otimes \Gamma)-\right.$ $\bar{L} C)^{T}$. Then, there always exist an integer $h \in \mathbb{N}^{+}$and a scalar $p_{2}\left(0<p_{2}<1\right)$ such that

$$
\|x(k+h)-\hat{x}(k+h)\|_{2} \leq p_{2}\|x(k)-\hat{x}(k)\|_{2} .
$$

In addition, the gain of state estimator for each node can be obtained by

$$
L_{i}=Q_{i+1}^{-1} \bar{L}_{i} .
$$

Proof: Denote $e_{e}(k) \triangleq x(k)-\hat{x}(k)$ and $F\left(e_{e}(k)\right) \triangleq$ $F(x(k))-F(\hat{x}(k))$. Then, in light of (14) and (15), the dynamics of estimation error is deduced as

$$
e_{e}(k+1)=F\left(e_{e}(k)\right)+((\mathcal{W} \otimes \Gamma)-L C) e_{e}(k) .
$$

By choosing the quadratic function $V_{2}(k)=$ $e_{e}(k)^{T} \mathcal{Q}_{2} e_{e}(k)$ and defining its difference as $\Delta V_{2}(k)=V_{2}(k+1)-V_{2}(k)$, we calculate the term $\Delta V_{2}(k)+\eta_{2} V_{2}(k)$ as

$$
\begin{aligned}
& \Delta V_{2}(k)+\eta_{2} V_{2}(k) \\
= & e_{e}^{T}(k+1) \mathcal{Q}_{2} e_{e}(k+1)-\left(1-\eta_{2}\right) e_{e}^{T}(k) \mathcal{Q}_{2} e_{e}(k) \\
= & e_{e}^{T}(k)\left[((\mathcal{W} \otimes \Gamma)-L C)^{T} \mathcal{Q}_{2}((\mathcal{W} \otimes \Gamma)-L C)\right. \\
& \left.-\left(1-\eta_{2}\right) \mathcal{Q}_{2}\right] e_{e}(k)+F^{T}\left(e_{e}(k)\right) \mathcal{Q}_{2} F\left(e_{e}(k)\right) \\
& +2 F^{T}\left(e_{e}(k)\right) \mathcal{Q}_{2}((\mathcal{W} \otimes \Gamma)-L C) e_{e}(k) .
\end{aligned}
$$

Similar to (34), it is easy to see from (2) that

$$
\varepsilon_{2}\left[\begin{array}{c}
e_{e}(k) \\
F\left(e_{e}(k)\right)
\end{array}\right]^{T}\left[\begin{array}{cc}
\hat{U}_{1} & -\hat{U}_{2} \\
* & I
\end{array}\right]\left[\begin{array}{c}
e_{e}(k) \\
F\left(e_{e}(k)\right)
\end{array}\right] \leq 0 .
$$

Subsequently, it can be obtained from (41) and (42) that

$$
\Delta V_{2}(k)+\eta_{2} V_{2}(k) \leq \xi^{T}(k) \bar{\Pi}_{2} \xi(k)
$$

where

$$
\bar{\Pi}_{2}=\left[\begin{array}{cc}
\bar{\Pi}_{21} & \bar{\Pi}_{22} \\
* & \mathcal{Q}_{2}-\varepsilon_{2} I
\end{array}\right]
$$

with $\bar{\Pi}_{21}=((\mathcal{W} \otimes \Gamma)-L C)^{T} \mathcal{Q}_{2}((\mathcal{W} \otimes \Gamma)-L C)-(1-$ $\left.\eta_{2}\right) \mathcal{Q}_{2}-\varepsilon_{2} \hat{U}_{1}$ and $\bar{\Pi}_{22}=((\mathcal{W} \otimes \Gamma)-L C)^{T} \mathcal{Q}_{2}+\varepsilon_{2} \hat{U}_{2}$.

By applying Schur Complement Lemma, we can acquire that $\Delta V_{2}(k)+\eta_{2} V_{2}(k) \leq 0$ in terms of (37) and (39), which implies that

$$
\begin{aligned}
V_{2}(k+h) & \leq\left(1-\eta_{2}\right) V_{2}(k+h-1) \\
& \leq\left(1-\eta_{2}\right)^{2} V_{2}(k+h-2) \leq \cdots \leq\left(1-\eta_{2}\right)^{h} V_{2}(k)
\end{aligned}
$$

Furthermore, we obtain

$$
\begin{aligned}
\underline{\lambda}_{\mathcal{Q}_{2}}\left\|e_{e}(k+h)\right\|_{2}^{2} & \leq\left(1-\eta_{2}\right) V_{2}(k+h) \\
\leq & \left(1-\eta_{2}\right)^{h} V_{2}(k) \leq\left(1-\eta_{2}\right)^{h} \bar{\lambda}_{\mathcal{Q}_{2}}\left\|e_{e}(k)\right\|_{2}^{2}
\end{aligned}
$$

where $\underline{\lambda}_{\mathcal{Q}_{2}} \triangleq \lambda_{\min }\left\{\mathcal{Q}_{2}\right\}$ and $\bar{\lambda}_{\mathcal{Q}_{2}} \triangleq \lambda_{\max }\left\{\mathcal{Q}_{2}\right\}$. To this end, we have $\|x(k+h)-\hat{x}(k+h)\|_{2}<p_{2}\|x(k)-\hat{x}(k)\|_{2}$, where $p_{2}=\sqrt{\rho_{2}}$ and $\rho_{2}=\left(1-\eta_{2}\right)^{h} \bar{\lambda}_{\mathcal{Q}_{2}} \underline{\lambda}_{\mathcal{Q}_{2}}^{-1}$. Thus, for $0<\eta_{2}<1$, there always exists an appropriate coding interval $h$ to guarantee $0<p_{2}<1$, and the proof is now complete.

\section{B. The analysis of cluster synchronization control}

The ultimate boundedness of the cluster synchronization error dynamics (16) is discussed in the following theorem.

Theorem 1: Under the bit rate constraints (6) and (7), let the positive integers $R_{s}, R_{\bar{i} i}(i \in \mathcal{V})$, and the matrix $K$ with appropriate dimensions be given. Then, the cluster synchronization error is ultimately bounded if there exist positive scalars $\varepsilon_{3}, \varepsilon_{4}, \theta$, and positive definite matrices $P_{i} \in \mathbb{R}^{n_{x} \times n_{x}}$ $(i \in \mathcal{V})$ such that

$$
\Pi_{3}=\left[\begin{array}{cccc}
\Pi_{3}^{1} & \varepsilon_{3} \hat{U}_{2} & 0 & \Pi_{3}^{2} \\
* & -\varepsilon_{3} I & 0 & \mathcal{P} \\
* & * & -\varepsilon_{4} I & K^{T} \mathcal{P} \\
* & * & * & -\mathcal{P}
\end{array}\right]<0
$$

with $\Pi_{3}^{1}=-(1-\theta) \mathcal{P}-\varepsilon_{3} \hat{U}_{1}, \Pi_{3}^{2}=(K+\mathcal{W} \otimes \Gamma)^{T} \mathcal{P}$, and $\mathcal{P}=\operatorname{diag}_{N}\left\{P_{i}\right\}$.

Proof: Define the Lyapunov-like function as $V(k)=$ $e^{T}(k) \mathcal{P} e(k)$. Then, the difference of $V(k)$ is calculated as:

$$
\begin{aligned}
\Delta V(k)= & V(k+1)-V(k) \\
= & e^{T}(k+1) \mathcal{P} e(k+1)-e^{T}(k) \mathcal{P} e(k) \\
= & {\left[F(e(k))+(K+\mathcal{W} \otimes \Gamma) e(k)+K d_{e}(k)\right]^{T} \mathcal{P} } \\
& \times\left[F(e(k))+(K+\mathcal{W} \otimes \Gamma) e(k)+K d_{e}(k)\right] \\
& -e^{T}(k) \mathcal{P} e(k) \\
\leq & {\left[F(e(k))+(K+\mathcal{W} \otimes \Gamma) e(k)+K d_{e}(k)\right]^{T} \mathcal{P} } \\
& \times\left[F(e(k))+(K+\mathcal{W} \otimes \Gamma) e(k)+K d_{e}(k)\right] \\
& -e^{T}(k) \mathcal{P} e(k) \\
& +\varepsilon_{3}\left[\begin{array}{c}
e(k) \\
F(e(k))
\end{array}\right]^{T}\left[\begin{array}{cc}
-\hat{U}_{1} & \hat{U}_{2} \\
* & -I
\end{array}\right]\left[\begin{array}{c}
e(k) \\
F(e(k))
\end{array}\right] \\
= & \zeta^{T}(k) \bar{\Pi}_{3} \zeta(k)-\theta V(k)+\varepsilon_{4} d_{e}^{T}(k) d_{e}(k)
\end{aligned}
$$

where

$$
\begin{aligned}
\zeta(k) & =\left[\begin{array}{lll}
e^{T}(k) & F^{T}(e(k)) & d_{e}^{T}(k)
\end{array}\right]^{T}, \\
\bar{\Pi}_{3} & =\left[\begin{array}{ccc}
\bar{\Pi}_{3}^{1} & \bar{\Pi}_{3}^{2} & (K+\mathcal{W} \otimes \Gamma)^{T} \mathcal{P} K \\
* & \mathcal{P}-\varepsilon_{3} I & \mathcal{P} K \\
* & * & K^{T} \mathcal{P} K-\varepsilon_{4} I
\end{array}\right], \\
\bar{\Pi}_{3}^{1} & =(K+\mathcal{W} \otimes \Gamma)^{T} \mathcal{P}(K+\mathcal{W} \otimes \Gamma)-(1-\theta) \mathcal{P}-\varepsilon_{3} \hat{U}_{1}, \\
\bar{\Pi}_{3}^{2} & =(K+\mathcal{W} \otimes \Gamma)^{T} \mathcal{P}+\varepsilon_{3} \hat{U}_{2} .
\end{aligned}
$$

By applying the Schur Complement Lemma, inequality (45) implies $\bar{\Pi}_{3}<0$. Then, it follows from (46) that

$$
\Delta V(k) \leq-\theta V(k)+\varepsilon_{4} d_{e}^{T}(k) d_{e}(k) .
$$

In addition, it follows immediately from Lemma 2 and the dynamics of $\breve{x}(d h)$ that

$$
\|x(d h)-\breve{x}(d h)\|_{2}^{2}
$$




$$
\begin{aligned}
\leq & \left\|x(d h)-\bar{x}(d h)-\hbar_{b(d h)}\left(s_{1}^{1}, \cdots, s_{n_{x}}^{N}\right)\right\|_{2}^{2} \\
\leq & \|x(d h)-\hat{x}(d h)\|_{2}^{2} \\
& +\left\|\hat{x}(d h)-\bar{x}(d h)-\hbar_{b(d h)}\left(s_{1}^{1}, \cdots, s_{n_{x}}^{N}\right)\right\|_{2}^{2} \\
\leq & 2 \rho_{2}^{d} N s_{0}+\sum_{i=1}^{N} \frac{n_{x} b_{i}^{2}}{\left(\left\lfloor\sqrt[n_{x}]{2^{R_{\bar{i}}}}\right\rfloor\right)^{2}}
\end{aligned}
$$

where $s_{0} \triangleq \sup _{x_{i 0} \in \mathcal{I}_{0}}\left\|x_{i 0}\right\|_{2}^{2}$, and $\rho_{2}$ is defined in Lemma 2 .

Notice that $\breve{x}(k)$ and $x(k)$ can be seen as two solutions of (14). Then, for $0<k<h$, we have $\|x(k)-\breve{x}(k)\|_{2}^{2} \leq$ $p_{1}^{k}\|x(0)-\breve{x}(0)\|_{2}^{2} \leq 2 p_{1}^{k} N s_{0}$ and, for $d h<k<(d+1) h$, we have $\|x(k)-\breve{x}(k)\|_{2}^{2} \leq p_{1}^{k-d h}\|x(d h)-\breve{x}(d h)\|_{2}^{2}$.

Denote $\bar{d} \triangleq\left\lfloor\frac{k}{h}\right\rfloor$ and $\bar{h} \triangleq \bmod (k, h)$. Then, it follows from $d_{e}(k)=\breve{x}(k)-x(k), p_{1} \geq 1$, and $0 \leq p_{2} \leq 1$ that

$$
\begin{aligned}
& d_{e}^{T}(k) d_{e}(k) \leq 2 p_{1}^{\bar{h}} \rho_{2}^{\bar{d}} N s_{0}+p_{1}^{\bar{h}} \sum_{i=1}^{N} \frac{n_{x} b_{i}^{2}}{\left(\left\lfloor\sqrt[n_{x}]{2^{R_{\bar{i} i}}}\right\rfloor\right)^{2}} \\
& \leq 2 p_{1}^{h} \rho_{2}^{\bar{d}} N s_{0}+p_{1}^{h} \sum_{i=1}^{N} \frac{n_{x} b_{i}^{2}}{\left(\left\lfloor\sqrt[n_{x}]{2^{R_{\bar{i}}}}\right\rfloor\right)^{2}} \\
& \leq 2 p_{1}^{h} N s_{0}+p_{1}^{h} \sum_{i=1}^{N} \frac{n_{x} b_{i}^{2}}{\left(\left[\sqrt[n_{x}]{2^{R_{\bar{i}}}}\right\rfloor\right)^{2}} .
\end{aligned}
$$

Denoting $\phi \triangleq 2 \varepsilon_{4} p_{1}^{h} N s_{0}+\varepsilon_{4} p_{1}^{h} \sum_{i=1}^{N} \frac{n_{x} b_{i}^{2}}{\left(\left[\sqrt[n_{x}]{2^{R_{\bar{i}}}}\right]\right)^{2}}$, it follows from (47) that

$$
\Delta V(k) \leq-\theta V(k)+\phi .
$$

Then, for any scalar $\eta_{3}$, we have

$$
\begin{aligned}
& \eta_{3}^{t+1} V(t+1)-\eta_{3}^{t} V(t) \\
= & \eta_{3}^{t+1}(V(t+1)-V(t))+\eta_{3}^{t}\left(\eta_{3}-1\right) V(t) \\
\leq & \eta_{3}^{t}\left(\eta_{3}-1-\eta_{3} \theta\right) V(t)+\eta_{3}^{t+1} \phi .
\end{aligned}
$$

Letting $\eta_{3}=\bar{\eta}_{3}=\frac{1}{1-\theta}$ and summing both sides of inequality (52) from 0 to $k-1$ in relation to $t$, we arrive at

$$
\bar{\eta}_{3}^{k} V(k)-V(0) \leq \frac{\bar{\eta}_{3}\left(1-\bar{\eta}_{3}^{k}\right)}{\left(1-\bar{\eta}_{3}\right)} \phi
$$

which can be further represented as the following form:

$$
\begin{aligned}
V(k) & \leq \frac{V(0)}{\bar{\eta}_{3}^{k}}+\frac{\bar{\eta}_{3}\left(1-\bar{\eta}_{3}^{k}\right)}{\bar{\eta}_{3}^{k}\left(1-\bar{\eta}_{3}\right)} \phi \\
& =(1-\theta)^{k}\left(V(0)-\frac{\phi}{\theta}\right)+\frac{\phi}{\theta} .
\end{aligned}
$$

Then, it follows from the definition of $V(k)$ and (54) that

$$
\begin{aligned}
\|e(k)\|_{2}^{2} & \leq \frac{1}{\lambda_{\min }\{\mathcal{P}\}} e^{T}(k) \mathcal{P} e(k) \\
& \leq \frac{(1-\theta)^{k}}{\lambda_{\min }\{\mathcal{P}\}}\left(V(0)-\frac{\phi}{\theta}\right)+\frac{\phi}{\theta \lambda_{\min }\{\mathcal{P}\}} .
\end{aligned}
$$

Consequently, it can be concluded from Definition 1 that the dynamics of the cluster synchronization error system (16) is exponentially ultimately bounded. The AUB of the cluster synchronization error can be computed as

$$
\frac{2 \varepsilon_{4} p_{1}^{h} N s_{0}+\varepsilon_{4} p_{1}^{h} \sum_{i=1}^{N} \frac{n_{x} b_{i}^{2}}{\left(\left\lfloor\sqrt[n_{x}]{2^{R_{\bar{i} i}}}\right\rfloor\right)^{2}}}{\theta \lambda_{\min }\{\mathcal{P}\}} .
$$

The proof of this theorem is now complete.

Next, the following bit rate condition, which can be readily established from (56), guarantees the desired cluster synchronization performance.

Corollary 1: Under the condition in Theorem 1, the cluster synchronization error system (16) is ultimately bounded with a given AUB $\epsilon$ if there exist a set of bit rates $R_{\bar{i} i}(i \in \mathcal{V})$ satisfying

$$
\sum_{i=1}^{N} \frac{n_{x} b_{i}^{2}}{\left(\left[\sqrt[n_{x}]{2^{R_{\bar{i} i}}}\right]\right)^{2}} \leq \frac{\theta \lambda_{\min }\{\mathcal{P}\} \epsilon-2 \varepsilon_{4} p_{1}^{h} N s_{0}}{\varepsilon_{4} p_{1}^{h}} .
$$

Specifically, when the allocated bit rate are all the same for each node, i.e., $R_{11}=R_{12}=\cdots=R_{m N} \triangleq \hat{R}$, the cluster synchronization error system (16) is ultimately bounded with a given AUB $\epsilon$ if

$$
\hat{R} \geq \frac{n_{x}}{2} \log _{2}\left(\frac{\varepsilon_{4} p_{1}^{h} n_{x} \sum_{i=1}^{N} b_{i}^{2}}{\theta \lambda_{\min }\{\mathcal{P}\} \epsilon-2 \varepsilon_{4} p_{1}^{h} N s_{0}}\right) .
$$

Remark 3: In Theorem 1, a sufficient condition is proposed under which the dynamics of the cluster synchronization error system (16) is ensured to be exponentially ultimately bounded. It is apparent that the AUB of the cluster synchronization error system (16) is dependent on the bound of the disturbance noise on the plant (1), the number of the nodes in the CDN, the coding period, and the coding-decoding procedure. In particular, when the bound of the noise, the number of nodes in the CDN, the coding period and the parameters of the codingdecoding procedure are all fixed, the AUB (56) of the cluster synchronization error system would be only dependent on the bit rate, thereby meriting the establishment of the bit rate condition under which the required cluster synchronization control performance is achieved.

\section{The design of cluster synchronization controller}

Based on the analysis of the cluster synchronization error system, we will now focus on the controller design issue by solving some optimization problems to be formulated by means of certain performance indices of interest.

$\boldsymbol{O P} \boldsymbol{A}$ : This optimization problem is to minimize the ultimate bound of the cluster synchronization error dynamics so as to achieve the best possible synchronization performance under bit rate constraints (6) and (7) with given total available bit rate $R_{s}$ and allocated bit rate $R_{\bar{i} i}$.

Theorem 2: For cluster synchronization error system (16), let a scalar $\bar{\theta}(0<\bar{\theta}<1)$ and positive integers $R_{s}, R_{\bar{i} i}$ $(i \in \mathcal{V})$ be given. Suppose that there exist positive scalars $\varepsilon_{3}, \varepsilon_{4}$, positive definite matrices $P_{i} \in \mathbb{R}^{n_{x} \times n_{x}}(i \in \mathcal{V})$, and 
real-valued matrices $\mathcal{K}_{i} \in \mathbb{R}^{n_{x} \times n_{x}}(i \in \mathcal{V})$ satisfying

$$
\Pi_{4}=\left[\begin{array}{cccc}
\Pi_{4}^{1} & \varepsilon_{3} \hat{U}_{2} & 0 & \Pi_{4}^{2} \\
* & -\varepsilon_{3} I & 0 & \mathcal{P} \\
* & * & -\varepsilon_{4} I & \mathcal{K}^{T} \\
* & * & * & -\mathcal{P}
\end{array}\right]<0
$$

with $\Pi_{4}^{1}=-\bar{\theta} \mathcal{P}-\varepsilon_{3} \hat{U}_{1}, \Pi_{4}^{2}=\mathcal{K}^{T}+(\mathcal{W} \otimes \Gamma)^{T} \mathcal{P}$, $\mathcal{K}=\operatorname{diag}_{N}\left\{\mathcal{K}_{i}\right\}$, and $\mathcal{P}=\operatorname{diag}_{N}\left\{P_{i}\right\}$. Then, the dynamics of the cluster synchronization error system (16) is ultimately bounded, where the decay rate is $\bar{\theta}$ and the minimum of the asymptotic bound of $\|e(k)\|_{2}^{2}$ can be obtained by solving the following minimization problem:

$$
\min \left\{2 \varepsilon_{4} p_{1}^{h} N s_{0}+\varepsilon_{4} p_{1}^{h} \sum_{i=1}^{N} \frac{n_{x} b_{i}^{2}}{\left(\left\lfloor\sqrt[n_{x}]{2^{R_{\bar{i}}}}\right\rfloor\right)^{2}}\right\}
$$

subject to the matrix inequality constraints (59)-(60). Moreover, the gains $K_{i}(i \in \mathcal{V})$ of the controller can be obtained from the following relationship:

$$
\operatorname{diag}_{N}\left\{K_{i}\right\}=\mathcal{P}^{-1} \mathcal{K} .
$$

Proof: Choosing $\theta=1-\bar{\theta}$, we conclude that

$$
\Pi_{4}^{1}=\Pi_{3}^{1}, \Pi_{4}^{2}=\Pi_{3}^{2} .
$$

Then, it follows from (59) that $\Pi_{3}<0$, which guarantees the ultimate boundedness of the cluster synchronization error system (16).

Along the similar line of the proof of Theorem 1, we obtain

$$
V(k) \leq \bar{\theta}^{k}\left(V(0)-\frac{\phi}{1-\bar{\theta}}\right)+\frac{\phi}{1-\bar{\theta}}
$$

where

$$
\phi=2 \varepsilon_{4} p_{1}^{h} N s_{0}+\varepsilon_{4} p_{1}^{h} \sum_{i=1}^{N} \frac{n_{x} b_{i}^{2}}{\left(\left\lfloor\sqrt[n_{x}]{2^{R_{\bar{i} i}}}\right\rfloor\right)^{2}} .
$$

Then, based on (55) and (60), it is readily seen that

$$
\|e(k)\|_{2}^{2} \leq V(k) \leq \bar{\theta}^{k}\left(V(0)-\frac{\phi}{1-\bar{\theta}}\right)+\frac{\phi}{1-\bar{\theta}} .
$$

By considering (55), the AUB of $\|e(k)\|_{2}^{2}$ can be calculated by minimizing $\phi$, which turns out to be equivalent to the condition (61). Then, the proof is complete.

$\boldsymbol{O P} \boldsymbol{B}$ : This optimization problem aims to maximize the decay rate of the cluster synchronization error dynamics for the fastest convergence under bit rate constraints (6)-(7) with given total available bit rate $R_{s}$ and allocated bit rate $R_{\bar{i} i}$.

Theorem 3: For cluster synchronization error system (16), let positive integers $R_{s}, R_{\bar{i} i}(i \in \mathcal{V})$ be given. Suppose that there exist positive scalars $\varepsilon_{3}, \varepsilon_{4}$, positive definite matrices $P_{i} \in \mathbb{R}^{n_{x} \times n_{x}}(i \in \mathcal{V}), \mathcal{R} \in \mathbb{R}^{N n_{x} \times N n_{x}}$, and real-valued matrices $\mathcal{K}_{i} \in \mathbb{R}^{n_{x} \times n_{x}}(i \in \mathcal{V})$ satisfying

$$
\Pi_{5}=\left[\begin{array}{cccc}
\Pi_{5}^{1} & \varepsilon_{3} \hat{U}_{2} & 0 & \Pi_{5}^{2} \\
* & -\varepsilon_{3} I & 0 & \mathcal{P} \\
* & * & -\varepsilon_{4} I & \mathcal{K}^{T} \\
* & * & * & -\mathcal{P}
\end{array}\right]<0
$$

$$
\begin{aligned}
& \mathcal{P} \geq I \\
& {\left[\begin{array}{cc}
-\mathcal{R} & \theta I \\
* & \mathcal{P}-2 I
\end{array}\right]<0 }
\end{aligned}
$$

with $\Pi_{5}^{1}=-\mathcal{P}+\mathcal{R}-\varepsilon_{3} \hat{U}_{1}, \Pi_{5}^{2}=\mathcal{K}^{T}+(\mathcal{W} \otimes \Gamma)^{T} \mathcal{P}$, $\mathcal{K}=\operatorname{diag}_{N}\left\{\mathcal{K}_{i}\right\}$, and $\mathcal{P}=\operatorname{diag}_{N}\left\{P_{i}\right\}$. Then, the dynamics of the cluster synchronization error system (16) is ultimately bounded, and the maximum decay rate of cluster synchronization error $\|e(k)\|_{2}^{2}$ can be computed by solving the following maximization problem:

$$
\max \{\theta\}
$$

subject to the constraints (65)-(67). Moreover, the gains $K_{i}$ $(i \in \mathcal{V})$ of the controller can be obtained from the following relationship:

$$
\operatorname{diag}_{N}\left\{K_{i}\right\}=P^{-1} \mathcal{K} .
$$

Proof: It can be concluded from the inequality

$$
(\mathcal{P}-I) \mathcal{P}^{-1}(\mathcal{P}-I) \geq 0
$$

that

$$
-\mathcal{P}^{-1} \leq \mathcal{P}-2 I .
$$

Then, it follows from (67) that

$$
\Pi_{5}^{3} \triangleq\left[\begin{array}{cc}
-\mathcal{R} & \theta I \\
* & -\mathcal{P}^{-1}
\end{array}\right] \leq\left[\begin{array}{cc}
-\mathcal{R} & \theta I \\
* & \mathcal{P}-2 I
\end{array}\right]<0 .
$$

By applying Schur Complement Lemma to $\Pi_{5}^{3}$, it can be obtained that $-\mathcal{R}+\theta^{2} \mathcal{P}<0$. Then, it follows from (65) that

$$
\left[\begin{array}{cccc}
\bar{\Pi}_{5}^{1} & \varepsilon_{3} \hat{U}_{2} & 0 & \Pi_{5}^{2} \\
* & -\varepsilon_{3} I & 0 & \mathcal{P} \\
* & * & -\varepsilon_{4} I & \mathcal{K}^{T} \\
* & * & * & -\mathcal{P}
\end{array}\right]<\Pi_{5}<0
$$

with $\bar{\Pi}_{5}^{1}=-\mathcal{P}+\theta^{2} \mathcal{P}-\varepsilon_{3} \hat{U}_{1}$. Then, it follows from Theorem 1 that the dynamics of the cluster synchronization error $e(k)$ is ultimately bounded.

Similar to the proof of Theorem 1, we obtain the following inequality:

$$
V(k) \leq\left(1-\theta^{2}\right)^{k}\left(V(0)-\frac{\phi}{\theta^{2}}\right)+\frac{\phi}{\theta^{2}}
$$

where

$$
\phi=2 \varepsilon_{4} p_{1}^{h} N s_{0}+\varepsilon_{4} p_{1}^{h} \sum_{i=1}^{N} \frac{n_{x} b_{i}^{2}}{\left(\left\lfloor\sqrt[n_{x}]{2^{R_{\bar{i}}}}\right\rfloor\right)^{2}},
$$

and the decay rate of $\|e(k)\|_{2}^{2}$ is thus determined by $1-\theta^{2}$. In this way, the maximum decay rate of cluster synchronization error is obtained by addressing the optimization problem (68), which completes the proof.

Remark 4: For cluster synchronization error systems, the ultimate bound and the decay rate are two essential performance indices. In order to achieve the improvements in different performance indices, $\boldsymbol{O P} \boldsymbol{A}$ and $\boldsymbol{O P} \boldsymbol{B}$ are proposed separately. The effect of each optimization problem will be shown numerically in IV section. 


\section{Co-design of controllers and bit rate allocation protocol}

With given bit rate $R_{s}$ and $R_{\bar{i} i}$, we have considered the design problem of cluster synchronization controllers in Theorem 2 with the aim of minimizing the AUB of the cluster synchronization error system (16). In fact, the available bit rate of each node plays an important role in the AUB, and it can be allocated by MAC protocol in the digital communication network. As such, we will now discuss the co-design problem in which both bit rate allocation protocol and controller gain serve as design parameters.

Based on the above discussions, we propose the following new minimization problem according to the bit-rate constraints (6) and (7).

Corollary 2: Based on Theorem 2, when the positive integers $R_{\bar{i} i}(i \in \mathcal{V})$ are variables to be determined, the minimum of the AUB of $\|e(k)\|_{2}^{2}$ can be derived by solving the following minimization problem:

$$
\begin{array}{ll}
\min & 2 \varepsilon_{4} p_{1}^{h} N s_{0}+\varepsilon_{4} p_{1}^{h} \sum_{i=1}^{N} \frac{n_{x} b_{i}^{2}}{\left(\left\lfloor\sqrt[n_{x}]{2^{R_{\bar{i} i}}}\right\rfloor\right)^{2}} \\
\text { s.t. } & (6),(7),(59),(60) \\
& 0 \leq R_{\bar{i} i} \leq R_{s} \\
& R_{\bar{i} i} \in \mathbb{N}, i \in \mathcal{V} .
\end{array}
$$

Moreover, the gains $K_{i}(i \in \mathcal{V})$ of controllers can be obtained from the relationship:

$$
\operatorname{diag}_{N}\left\{K_{i}\right\}=P^{-1} \mathcal{K}
$$

Proof: The proof is similar to Theorem 2, and is therefore omitted for the sake of brevity.

It is easily observed that the objective function and constraints of $\boldsymbol{O P} \boldsymbol{A}$ in Theorem 2 are linear and can thus be resolved efficiently by the linear matrix inequality (LMI) technique. The co-design problem presented in this subsection is actually a mixed-integer nonlinear programming (MINP) problem, which is difficult to solve due to the integer constraints of $R_{\bar{i}}$, the matrix inequality constraints (59) and (60), in addition to the presence of the nonlinear term

$$
\varepsilon_{4} p_{1}^{h} \sum_{i=1}^{N} \frac{n_{x} b_{i}^{2}}{\left(\left\lfloor\sqrt[n_{x}]{2^{R_{\bar{i} i}}}\right\rfloor\right)^{2}}
$$

in the objective function.

To deal with the emerging MINP problem, the particle swarm optimization (PSO) algorithm and the LMI technique are employed together in this paper. In order to take full advantage of the PSO algorithm, a suitable objective function should be given for the proposed optimization problem. As such, the optimization problem (73) is transformed into the following form by introducing a penalty function:

$$
\begin{array}{ll}
\min & 2 \varepsilon_{4} p_{1}^{h} N s_{0}+\varepsilon_{4} p_{1}^{h} \sum_{i=1}^{N} \frac{n_{x} b_{i}^{2}}{\left(\left\lfloor\sqrt[n_{x}]{2^{R_{\bar{i}}}}\right\rfloor\right)^{2}} \\
& +f_{p c}(R)+f_{p n}(\bar{R}) \\
\text { s.t. } & (59),(60) \\
& R_{\bar{i} i} \in \mathbb{N}, i \in \mathcal{V} .
\end{array}
$$

where

$$
f_{p c}(R)=\max \left\{0, \sum_{l=1}^{m} R_{l}-R_{s}\right\}
$$

is the penalty function with $R=\left[R_{1}, R_{2}, \cdots, R_{m}\right]$, and

$$
f_{p n}(\bar{R})=\sum_{\bar{i}=1}^{m}\left(\max \left\{0, \sum_{i \in \mathcal{V}_{\bar{i}}} R_{\bar{i} i}-R_{\bar{i}}\right\}\right)
$$

is the penalty function with $\bar{R}=\left[R_{\overline{1} 1}, R_{\overline{2} 2}, \cdots, R_{\bar{N} N}\right]$. The fitness function of the PSO algorithm is defined as

$$
\begin{aligned}
\mathbf{F}(R, \bar{R}) \triangleq & 2 \varepsilon_{4} p_{1}^{h} N s_{0}+\varepsilon_{4} p_{1}^{h} \sum_{i=1}^{N} \frac{n_{x} b_{i}^{2}}{\left(\left\lfloor\sqrt[n_{x}]{2^{R_{\bar{i} i}}}\right\rfloor\right)^{2}} \\
& +f_{p c}(R)+f_{p n}(\bar{R}) .
\end{aligned}
$$

\section{Algorithm 1 PSO-Assisted the Co-design Algorithm \\ - Step 1. Initialize the parameters of PSO: Initialize parameters $\mathbf{N}_{S}, \mathbf{N}_{I}, \mathbf{c}_{1}, \mathbf{c}_{2}, \mathbf{w}$, and the initial velocity $\mathbf{V}_{i}$ and position $\mathbf{R}_{i}$ of each particle. \\ - Step 2. Evaluate the fitness and set initial $\mathrm{p}_{i}$ : Evaluate} each particle's fitness function $\mathbf{F}\left(\mathbf{R}_{i}\right)$ by solving the LMIs (59) and (60). If the LMIs (59) and (60) are infeasible, then the value of fitness will be artificially assigned a sufficiently large value $\left(10^{4}\right.$ in this paper) to reduce the effect of the corresponding particle on the particle swarm. Set all initial positions as $\mathbf{p}_{i}$, $i=1,2, \cdots, \mathbf{N}_{s}$.

- Step 3. Select $g_{b}$ in the swarm: Select the $g_{b}$ in the swarm, which has the minimum fitness value.

- Step 4. Update the particle's velocity and position: Update the velocity and the position of the each particle by given updating equations (76) and (77).

- Step 5. Evaluate the fitness and update $\mathrm{p}_{i}$ : Evaluate each updated particle's fitness function by the same method in Step 2. If $\mathbf{F}\left(\mathbf{R}_{i}\right)<\mathbf{F}\left(\mathbf{p}_{i}\right)$, then, set the current position as $\mathbf{p}_{i}$.

- Step 6. Update $g_{b}$ in the swarm: Select the $g_{b}$ in the swarm, which has the minimum fitness value.

- Step 7. Design of the bit rate allocation protocol: Each iteration repeats the process of steps 4 and 6 until the maximum number of iterations is reached. Then, select the $g_{b}$ as the parameters of bit rate allocation protocol.

- Step 8. Design of the cluster synchronization controllers: Produce the synchronization controllers (10) by using the gain matrices $K_{i}$ obtained according to the selected bit rate protocol.

The PSO algorithm is outlined in Algorithm 1, where $\mathbf{N}_{S}$ denotes the population size, $\mathbf{N}_{I}$ stands for the maximum number of iterations, and $\mathbf{v}_{i}=\left[\mathbf{v}_{i, 1}, \mathbf{v}_{i, 2}, \cdots, \mathbf{v}_{i, m+N}\right]$ and $\mathbf{R}_{i}=\left[\mathbf{R}_{i, 1}, \mathbf{R}_{i, 2}, \cdots, \mathbf{R}_{i, m+N}\right]$ represent the velocity and position of particle $i$, respectively. The velocity and position updating equations of particle $i$ are given as follows:

$$
\begin{aligned}
\mathbf{v}_{i}(t+1)= & \mathbf{w} \mathbf{v}_{i}(t)+\mathbf{c}_{1} \mathbf{r}_{1}\left(\mathbf{p}_{i}(t)-\mathbf{R}_{i}(t)\right) \\
& +\mathbf{c}_{2} \mathbf{r}_{2}\left(g_{b}(t)-\mathbf{R}_{i}(t)\right)
\end{aligned}
$$




$$
\mathbf{R}_{i}(t+1)=\mathbf{R}_{i}(t)+\mathbf{v}_{i}(t+1)
$$

where $\mathbf{p}_{i}$ denotes the historical individual best position for particle $i, g_{b}$ bespeaks the historical global best position for the entire swarm, $\mathbf{w}$ is the inertia weight, $\mathbf{c}_{1}$ and $\mathbf{c}_{2}$ indicate the cognitive acceleration coefficient and the social acceleration coefficient, separately, and $t$ refers to the iteration number. In terms of the characteristic of integer variables, the initial position and velocity of the particle, as well as the parameters $\mathbf{c}_{1}, \mathbf{c}_{2}$, and $\mathbf{w}$ are selected as integers in the algorithm. Moreover, $\mathbf{r}_{1}$ and $\mathbf{r}_{2}$ are selected randomly from two integers 1 or 2.

Remark 5: In practical networks, the bit rate allocation protocol is a parameter that can be designed according to different performance specifications. On the other hand, each node's bit rate plays a significant role in improving the cluster synchronization performance. Therefore, the co-design problem of integrating the bit rate protocol and the controller parameters is considered in this paper. Furthermore, such a codesign problem is formulated as a MINP problem represented by (75), which can be well solved by Algorithm 1. One of our further research topics is to solve the MINP problem by using some effective optimization methods [23], [24].

Remark 6: In this paper, the cluster synchronization control problem is investigated for CDNs. A bit rate model with two constraints is first introduced to reflect the bandwidth limitation of CDNs with $m$ clusters. A sufficient condition is proposed in Theorem 1 to guarantee the ultimate boundedness of the cluster synchronization error dynamics. Two essential performance indices for the dynamics of the cluster synchronization error (i.e., the ultimate bound and the decay rate) are reflected in Theorem 2 and Theorem 3 by designing appropriate controller gains, respectively. To further reduce the ultimate bound, a co-design problem is proposed that comprehensively considers the bit rate allocation protocol and controller parameters, and such a problem is solved in Corollary 2.

Remark 7: The synchronization control problem for discrete-time complex dynamical networks has received considerable research interest from various communities and a rich body of literature has been available. In comparison with the existing studies, the main results of this paper exhibit the following distinctive contributions: 1) the bit rate model established in this paper is new that quantifies the communication bandwidth constraints and the corresponding bandwidth allocation protocols; rules for the clusters within the $\mathrm{CDN}$ and for the nodes within a cluster; 2) the proposed bit rate condition is new that discovers the relationship between the bit rate and the specific synchronization performance; and 3) the two formulated optimization problems and the associated co-design problem are new that reflect the synchronization performance indices.

\section{Numerical ExAmple}

This section presents three simulation scenarios to demonstrate the effectiveness of the decoder-based cluster synchronization controller proposed in this paper for the CDN (1).
The CDN (1) with five nodes is divided into two clusters $\mathcal{V}_{1}=\{1,2\}$ and $\mathcal{V}_{2}=\{3,4,5\}$. The coupling configuration matrix is of the following form:

$$
\mathcal{W}=\left[\begin{array}{ccccc}
-0.2 & 0.2 & 0.1 & -0.1 & 0 \\
0.2 & -0.2 & -0.1 & 0.1 & 0 \\
0.1 & -0.1 & -0.5 & 0.3 & 0.2 \\
-0.1 & 0.1 & 0.3 & -0.4 & 0.1 \\
0 & 0 & 0.2 & 0.1 & -0.3
\end{array}\right]
$$

and the inner-coupling matrix is an identity diagonal matrix.

The nonlinear functions of nodes in the two clusters respectively satisfy the following forms:

$$
\begin{aligned}
& f_{1}\left(x_{i}(k)\right)=\left[\begin{array}{l}
0.6 \tanh \left(0.1 x_{i 1}(k)\right)+1.05 x_{i 1}(k) \\
0.6 \tanh \left(0.1 x_{i 2}(k)\right)+1.05 x_{i 2}(k)
\end{array}\right] \\
& f_{2}\left(x_{i}(k)\right)=\left[\begin{array}{l}
0.8 \tanh \left(0.1 x_{i 1}(k)\right)+1.05 x_{i 1}(k) \\
0.8 \tanh \left(0.1 x_{i 2}(k)\right)+1.05 x_{i 2}(k)
\end{array}\right] .
\end{aligned}
$$

Then, it can be seen that the nonlinear functions satisfy the sector bounded condition (2) with

$$
\begin{aligned}
& \bar{u}_{1}=\left[\begin{array}{cc}
1.11 & 0 \\
0 & 1.11
\end{array}\right], \underline{u}_{1}=\left[\begin{array}{cc}
1.05 & 0 \\
0 & 1.05
\end{array}\right] \\
& \bar{u}_{2}=\left[\begin{array}{cc}
1.13 & 0 \\
0 & 1.13
\end{array}\right], \underline{u}_{2}=\left[\begin{array}{cc}
1.05 & 0 \\
0 & 1.05
\end{array}\right] .
\end{aligned}
$$

The measurements of the CDN (1) are modeled by the following parameters:

$$
\begin{aligned}
& C_{1}=\left[\begin{array}{ll}
1 & 1
\end{array}\right], C_{2}=\left[\begin{array}{ll}
1 & -0.90
\end{array}\right], C_{3}=\left[\begin{array}{ll}
0.80 & 0.60
\end{array}\right] \\
& C_{4}=\left[\begin{array}{ll}
0.70 & 1
\end{array}\right], C_{5}=\left[\begin{array}{ll}
1 & 1
\end{array}\right] .
\end{aligned}
$$

The bound of the initial value $s_{0}$ is set to be 4 . Then, the initial values of the target trajectories $t_{1}(k)$ and $t_{2}(k)$ are set to $\left[\begin{array}{ll}0.9 & -0.9\end{array}\right]^{T}$ and $\left[\begin{array}{ll}0.8 & -0.8\end{array}\right]^{T}$, the initial states of $x_{1}, x_{2}$, $x_{3}, x_{4}$ and $x_{5}$ are chosen as $\left[\begin{array}{ll}0.1 & -0.1\end{array}\right]^{T},\left[\begin{array}{ll}0.2 & -0.2\end{array}\right]^{T}$, $\left[\begin{array}{ll}0.1 & -0.1\end{array}\right]^{T},\left[\begin{array}{ll}0.2 & -0.2\end{array}\right]^{T}$, and $\left[\begin{array}{ll}0.3 & -0.3\end{array}\right]^{T}$, and the initial state of each estimator is set to zero. Next, we will verify the results of this paper through the following three cases.

Case 1: Cluster synchronization effects of $\boldsymbol{O P} \boldsymbol{A}$ and $\boldsymbol{O P} \boldsymbol{B}$.

In this case, the effects of $\boldsymbol{O P} \boldsymbol{A}$ and $\boldsymbol{O P} \boldsymbol{B}$ on different performances of cluster synchronization control are discussed. The total available bit rate of the digital communication network is assumed to be $16 \mathrm{bps}$, the available bit rate of each cluster is allocated by an average allocation protocol (AAP) as $R_{1}=R_{2}=8 \mathrm{bps}$, and the available bit rate of each node is allocated as $R_{11}=R_{12}=4, R_{23}=R_{24}=3, R_{25}=2$. The scaling parameters of each quantizer are chosen as $b_{1}=1$, $b_{2}=0.8, b_{3}=0.5, b_{4}=1.2, b_{5}=2$. The $\bar{\theta}$ is set to be 0.92 for $\boldsymbol{O P} \boldsymbol{A}$. Then, by applying Theorem 2 and Theorem 3, the controller gains of each node can be obtained, respectively.

The corresponding simulation results are depicted in Figs. 24 and Table I, where Fig. 2 describes the trajectories of the uncontrolled dynamical nodes, Fig. 3 depicts the trajectories of the controlled dynamical nodes where the controllers are derived by solving $\boldsymbol{O P} \boldsymbol{A}$, and Fig. 4 plots the evolutions of 
TABLE I

THE COMPARISON BETWEEN $\boldsymbol{O P} \boldsymbol{A}$ AND $\boldsymbol{O P} \boldsymbol{B}$

\begin{tabular}{rcc}
\hline Total bit rate $R_{s}(\mathrm{bps})$ & OP A $(\bar{\theta}=0.92)$ & OP $\mathbf{B}$ \\
\hline Settling-like time & 3 & 2 \\
\hline Upper bound of the trajectory & 1.2847 & 2.0381 \\
\hline \hline
\end{tabular}

the cluster synchronization error dynamics in the case of $\boldsymbol{O P}$ $\boldsymbol{A}$ and $\boldsymbol{O P} \boldsymbol{B}$. The AUB and the "settling-like times" (the time required for the cluster synchronization dynamics to reach and remain within the "steady-state region") of the cluster synchronization error dynamics with respect to $\boldsymbol{O P} \boldsymbol{A}$ and $\boldsymbol{O P}$ $\boldsymbol{B}$ are shown in Table I. From Figs. 2-3, it can be readily observed that the controllers obtained by solving $\boldsymbol{O P} \boldsymbol{A}$ are able to drive the CDN to the selected cluster synchronization pattern. Table I shows that $\boldsymbol{O P} \boldsymbol{A}$ result in a small AUB and the $\boldsymbol{O P} \boldsymbol{B}$ make for a low decay rate.

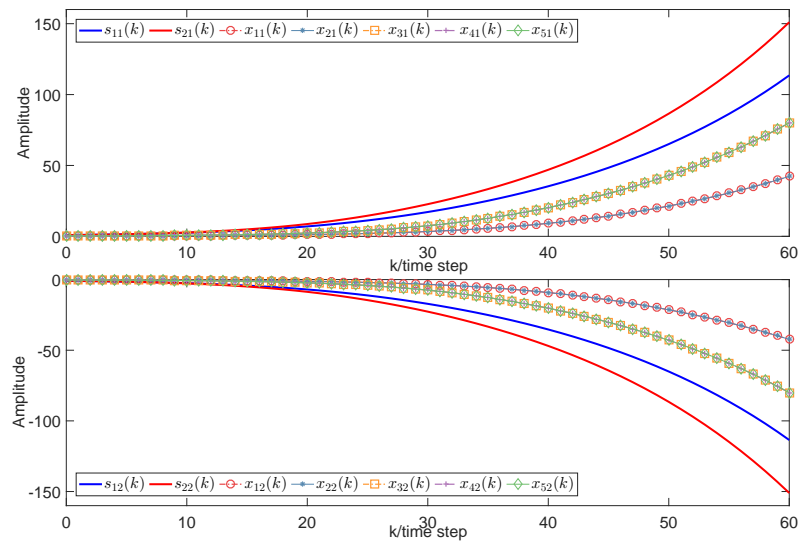

Fig. 2. The trajectories $x_{i j}(k)$ of uncontrolled nodes $i(i \in\{1,2,3,4,5\}$, $j \in\{1,2\})$
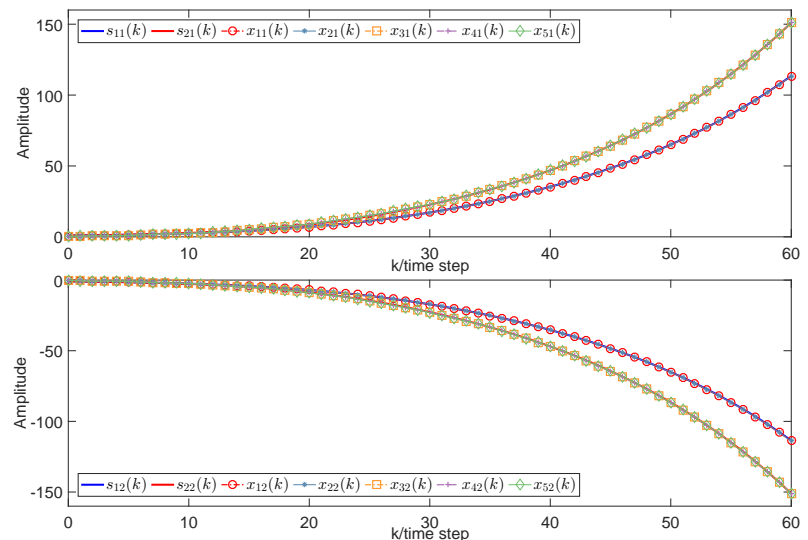

Fig. 3. The trajectories $x_{i j}(k)$ of controlled nodes $i(i \in\{1,2,3,4,5\}$, $j \in\{1,2\})$

Case 2: Effects of different allocation protocols on AUB.

In Case 1, the bit rate allocation protocol is assumed to be AAP, which allocates the total bit rate evenly to each node regardless of each node's characteristics. In comparison, the bit rate allocation protocol in Corollary 2 is calculated by the PSO-assisted the co-design algorithm. The bit rate

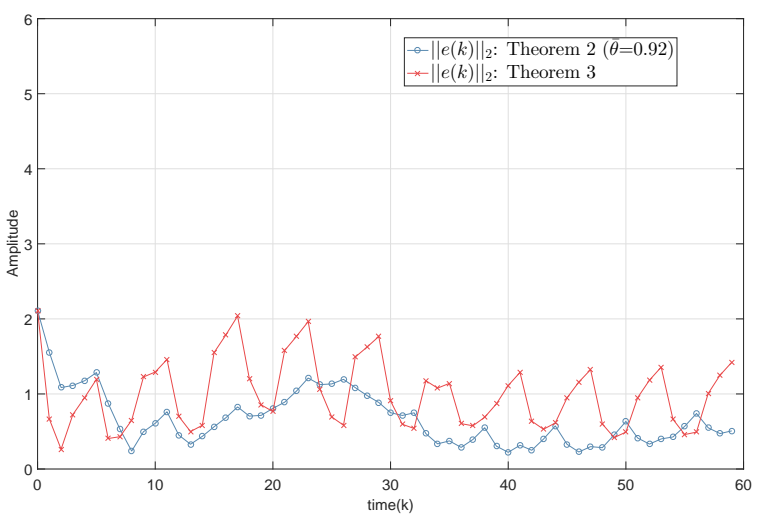

Fig. 4. The trajectories of cluster synchronization error $\|e(k)\|_{2}$ subject to $\boldsymbol{O P} \boldsymbol{A}$ and $\boldsymbol{O P} \boldsymbol{B}$

allocation protocol obtained in Corollary 2 can be referred to as PSO-based optimal allocation protocol (PSO-OAP), which allocates a specific bit rate to each node with the objective of minimizing AUB. This case aims to show the superiority of PSO-OAP over AAP in reducing AUB.

The scaling parameters are selected as $b_{1}=1, b_{2}=0.8$, $b_{3}=0.5, b_{4}=1.2$, and $b_{5}=2$. Two sets of simulations are conducted for Theorem 2 and Corollary 2, respectively, with the same total available bit rate of 32 bps. The decay rate $\bar{\theta}$ in Theorem 2 is set to be 0.92 . Then, the first set of simulations is conducted without considering the bit rate constraint on the cluster. While the second set of simulations is carried out in case the two clusters are equally allocated a certain bit rate, i.e., $R_{1}=R_{2}=16$ bps. Then, the AUB's values can be obtained by solving $\boldsymbol{O P} \boldsymbol{A}$ and MINP problem (73), respectively. The corresponding results are shown in Table II, where the first two rows outline the results of the first set simulations, and the last two rows present the results of the second set simulations. From Table II, it can be easily seen that PSO-OAP is capable of reducing the AUB of the cluster synchronization error system.

TABLE II

THE AUBS SUBJECT TO DIFFERENT BIT RATE ALLOCATION PROTOCOLS

\begin{tabular}{cccc}
\hline \hline Quantizer Parameters & Protocol & Allocation of Bit Rate & AUB \\
\hline & & $R_{11}=R_{12}=6$ & \\
$b_{1}=1, b_{2}=0.8$ & AAP & $R_{23}=R_{24}=6$ & 9.4906 \\
$b_{3}=0.5, b_{4}=1.2$ & & $R_{25}=6$ & \\
$b_{5}=2$ & \multirow{3}{*}{$R_{11}=7, R_{12}=6$} \\
& PSO-OAP & $R_{23}=5, R_{24}=6$ & 9.4454 \\
& & $R_{25}=8$ & \\
\cline { 2 - 4 } & & $R_{11}=R_{12}=8$ \\
$b_{1}=1, b_{2}=0.8$ & AAP & $R_{23}=R_{24}=5$ & 9.5112 \\
$b_{3}=0.5, b_{4}=1.2$ & & $R_{25}=6$ & \\
\cline { 2 - 4 }$b_{5}=2$ & & $R_{11}=8, R_{12}=8$ \\
& PSO-OAP & $R_{23}=4, R_{24}=6$ & 9.4836 \\
& \multicolumn{3}{c}{$R_{25}=6$} \\
\hline \hline
\end{tabular}

Case 3: Effects of total available bit rate on AUB and decay rate.

Intuitively, the bit rate has a significant impact on the cluster 
TABLE III

THE AUBS AND DECAY RATES SUBJECT TO DIFFERENT TOTAL AVAILABLE BIT RATES

\begin{tabular}{cccccc}
\hline \hline \multicolumn{2}{c}{ Total bit rate $R_{s}(\mathrm{bps})$} & 16 & 32 & 64 & 128 \\
\hline \multirow{2}{*}{ OP A } & $\begin{array}{c}\text { AUB } \psi \\
(\bar{\theta}=0.7)\end{array}$ & 16.115 & 15.2678 & 15.1683 & 15.1671 \\
\hline \multirow{2}{*}{ OP B } & AUB $\psi$ & 25.1405 & 23.8187 & 23.6635 & 23.6616 \\
\cline { 2 - 6 } & Decay rate $\theta$ & 0.7618 & 0.7618 & 0.7618 & 0.7618 \\
\hline \hline
\end{tabular}

synchronization performance. This case seeks to examine numerically the effects of different total available bit rate on the AUB and decay rate. The total bit rate is assumed to be 16 bps, 32 bps, 64 bps, and 128 bps, respectively. The scaling parameters of quantizers are set to be $b_{1}=8, b_{2}=9, b_{3}=8$, $b_{4}=7$. The $\bar{\theta}$ is set to be 0.7 for $\boldsymbol{O P} \boldsymbol{A}$. Then, under the AAP, we can derive the AUB and the decay rate by solving $\boldsymbol{O P} \boldsymbol{A}$ and $\boldsymbol{O P} \boldsymbol{B}$. The results are displayed in Table III.

It can be observed from Table III that the AUB decreases as the total bit rate increases, whether for $\boldsymbol{O P} \boldsymbol{A}$ or $\boldsymbol{O P} \boldsymbol{B}$. The advantage of $\boldsymbol{O P} \boldsymbol{A}$ over $\boldsymbol{O P} \boldsymbol{B}$ in reducing the AUB can also be easily verified. On the other hand, the decay rate is not affected by the total bit rate in $\boldsymbol{O P} \boldsymbol{B}$.

\section{CONCLUSiOnS}

In this paper, the cluster synchronization control problem has been investigated for discrete-time CDNs with constrained bit rate. A bit rate constraint model has been proposed to describe the bandwidth limit of a CDN with $m$ clusters. A sufficient condition has been proposed under which the cluster synchronization error system is ultimately bounded, and a bit rate condition that guarantees a certain cluster synchronization performance has been proposed subsequently. Two OPs have been presented and solved to calculate the required cluster synchronization controllers so as to achieve two different synchronization performance indices. The co-design issue of the bit rate allocation protocol and controller gains has been discussed as well. Three illustrative numerical cases have been presented to demonstrate the feasibility and the effectiveness of the proposed synchronization control strategies. Finally, the research idea of analyzing the impact of constrained bit rate on system performance can be extended to the filtering and control of networked systems [10], [26], [53], [54], [56].

\section{REFERENCES}

[1] A. S. Althobaiti and M. Abdullah, Medium access control protocols for wireless sensor networks classifications and cross-layering, Procedia Computer Science, vol. 65, pp. 4-16, 2015.

[2] S. Boccaletti, V. Latora, Y. Moreno, M. Chavez, and D. Hwang, Complex networks : Structure and dynamics, Physics Reports, vol. 424, pp. 175-308, 2006.

[3] J. Cao and L. Li, Cluster synchronization in an array of hybrid coupled neural networks with delay, Neural Networks, vol. 22, no. 4, pp. 335$342,2009$.

[4] J. Chen and Q. Ling, Bit-rate conditions for the consensus of quantized multiagent systems with network-induced delays based on event triggering, IEEE Transactions on Cybernetics, vol. 51, no. 2, pp. 984-993, 2021.
[5] Y. Chen, Z. Wang, J. Hu and Q.-L. Han, Synchronization control for discrete-time-delayed dynamical networks with switching topology under actuator saturations, IEEE Transactions on Neural Networks and Learning Systems, vol. 32, no. 5, pp. 2040-2053, 2021.

[6] Y. Chen, Z. Wang, B. Shen and H. Dong, Exponential synchronization for delayed dynamical networks via intermittent control: Dealing with actuator saturations, IEEE Transactions on Neural Networks and Learning Systems, vol. 30, no. 4, pp. 1000-1012, 2019.

[7] A. Farhadi and C. D. Charalambous, Stability and reliable data reconstruction of uncertain dynamic systems over finite capacity channels, Automatica, vol. 46, no. 5, pp. 889-896, 2010.

[8] N. Hou, H. Dong, Z. Wang and H. Liu, A partial-nodes-based approach to state estimation for complex networks with sensor saturations under random access protocol, IEEE Transactions on Neural Networks and Learning Systems, in press, DOI: 10.1109/TNNLS.2020.3027252.

[9] N. Hou, Z. Wang, D. W. C. Ho and H. Dong, Robust partial-nodesbased state estimation for complex networks under deception attacks, IEEE Transactions on Cybernetics, vol. 50, no. 6, pp. 2793-2802, 2020.

[10] J. Hu, H. Zhang, H. Liu and X. Yu, A survey on sliding mode control for networked control systems, International Journal of Systems Science, vol. 52, no. 6, pp. 1129-1147, 2021.

[11] J. Hu, Z. Wang, G.-P. Liu and H. Zhang, Variance-constrained recursive state estimation for time-varying complex networks with quantized measurements and uncertain inner coupling, IEEE Transactions on Neural Networks and Learning Systems, vol. 31, no. 6, pp. 1955-1967, 2020.

[12] T. Huang, C. Li, and X. Liu, Synchronization of chaotic systems with delay using intermittent linear state feedback, Chaos, vol. 18 , art. no. $033122,2008$.

[13] K. Kaneko, Relevance of dynamic clustering to biological networks, Physica D: Nonlinear Phenomena, vol. 75, no. 1-3, pp. 55-73, 1994.

[14] B. Li, Z. Wang, Q.-L. Han and H. Liu, Distributed quasiconsensus control for stochastic multiagent systems under Round-Robin protocol and uniform quantization, IEEE Transactions on Cybernetics, in press, DOI: $10.1109 /$ TCYB.2020.3026001

[15] J. Li, Z. Wang, H. Dong and X. Yi, Outlier-resistant observer-based control for a class of networked systems under coding-decoding mechanism, IEEE Systems Journal, in press, DOI: 10.1109/JSYST.2020.3044238.

[16] Q. Li, Z. Wang, W. Sheng, F. E. Alsaadi and F. E. Alsaadi, Dynamic event-triggered mechanism for $H_{\infty}$ non-Fragile state estimation of complex networks under randomly occurring sensor saturations, Information Sciences, vol. 509, pp. 304-316, 2020.

[17] T. Li, M. Fu, L. Xie, and J. F. Zhang, Distributed consensus with limited communication data rate, IEEE Transactions on Automatic Control, vol. 56, no. 2, pp. 279-292, 2011.

[18] T. Li and L. Xie, Distributed consensus over digital networks with limited bandwidth and time-varying topologies, Automatica, vol. 47, no. 9, pp. 2006-2015, 2011.

[19] Q. Liu and Z. Wang, Moving-horizon estimation for linear dynamic networks with binary encoding schemes, IEEE Transactions on Automatic Control, vol. 66, no. 4, pp. 1763-1770, 2021.

[20] S. Liu, Z. Wang, L. Wang and G. Wei, $H_{\infty}$ pinning control of complex dynamical networks under dynamic quantization effects: A coupled backward Riccati equation approach, IEEE Transactions on Cybernetics, in press, DOI: 10.1109/TCYB.2020.3021982.

[21] X. Liu and T. Chen, Cluster synchronization in directed networks via intermittent pinning control, IEEE Transactions on Neural Networks, vol. 22, no. 7, pp. 1009-1020, 2011.

[22] Y. Liu, Z. Wang, Y. Yuan and W. Liu, Event-triggered partial-nodesbased state estimation for delayed complex networks with bounded distributed delays, IEEE Transactions on Systems, Man, and Cybernetics: Systems, vol. 49, no. 6, pp. 1088-1098, Jun. 2019.

[23] Y. Liu, Q. Cheng, Y. Gan, Y. Wang, Z. Li and J. Zhao, Multi-objective optimization of energy consumption in crude oil pipeline transportation system operation based on exergy loss analysis, Neurocomputing, vol. 332, pp. 100-110, Mar. 2019.

[24] Y. Liu, S. Chen, B. Guan and P. Xu, Layout optimization of large-scale oil-gas gathering system based on combined optimization strategy, Neurocomputing, vol. 332, pp. 159-183, Mar. 2019.

[25] Y. C. Liu and N. Chopra, Robust controlled synchronization of interconnected robotic systems, in Proceedings of the 2010 American Control Conference, ACC 2010, pp. 1434-1439, Baltimore, MD, USA, 2010.

[26] J. Mao, Y. Sun, X. Yi, H. Liu and D. Ding, Recursive filtering of networked nonlinear systems: A survey, International Journal of Systems Science, vol. 52, no. 6, pp. 1110-1128, 2021. 
[27] G. N. Nair and R. J. Evans, Stabilizability of stochastic linear systems with finite feedback data rates, SIAM Jour. Control \& Optimization, vol. 43, no. 2, pp. 413-436, 2004.

[28] H. Nijmeijer and I. M. Mareels, An observer looks at synchronization, IEEE Transactions on Circuits and Systems I: Fundamental Theory and Applications, vol. 44, no. 10, pp. 882-890, 1997.

[29] H. Rao, Y. Xu, H. Peng, R. Lu, and C.-Y. Su, Quasi-synchronization of time delay Markovian jump neural networks with impulsive driven transmission and fading channels, IEEE Transactions on Cybernetics, vol. 50, no. 9, pp. 4121-4131, 2020.

[30] I. Rhee, A. Warrier, M. Aia, J. Min and M. L. Sichitiu, Z-MAC: A hybrid MAC for wireless sensor networks, IEEE/ACM Transactions on Networking, vol. 16, no. 3, pp. 511-524, 2008.

[31] M. G. Rosenblum, A. S. Pikovsky, and J. Kurths, Phase synchronization of chaotic oscillators, Physical Review Letters, vol. 76, no. 11, pp. 1804-1807, 1996.

[32] N. F. Rulkov, Images of synchronized chaos: Experiments with circuits, Chaos: An Interdisciplinary Journal of Nonlinear Science, vol. 6, no. 3, pp. 262-279, 1996.

[33] B. Shen, Z. Wang, D. Wang and Q. Li, State-saturated recursive filter design for stochastic time-varying nonlinear complex networks under deception attacks, IEEE Transactions on Neural Networks and Learning Systems, vol. 31, no. 10, pp. 3788-3800, 2020.

[34] S. H. Strogatz, Exploring complex networks, Nature, vol. 410, no. 6825, pp. 268-276, 2001.

[35] H. Su, Z. Rong, M. Z. Chen, X. Wang, G. Chen, and H. Wang, Decentralized adaptive pinning control for cluster synchronization of complex dynamical networks, IEEE Transactions on Cybernetics, vol. 43, no. 1, pp. 394-399, 2013.

[36] J. Suo, Z. Wang and B. Shen, Pinning synchronization control for a class of discrete-time switched stochastic complex networks under event-triggered mechanism, Nonlinear Analysis: Hybrid Systems, vol. 37, art. no. 100886, 2020.

[37] S. Tatikonda and S. Mitter, Control under communication constraints, IEEE Transactions on Automatic Control, vol. 49, no. 7, pp. 10561068, 2004.

[38] K. Wang, X. Fu, and K. Li, Cluster synchronization in community networks with nonidentical nodes, Chaos, vol. 19, art. no. 023106 , 2009.

[39] L. Wang, Z. Wang, B. Shen and G. Wei, Recursive filtering with measurement fading: A multiple description coding scheme, IEEE Transactions on Automatic Control, in press, DOI: 10.1109/TAC. 2020.3034196

[40] L. Wang, Z. Wang, G. Wei and F. E. Alsaadi, Observer-based consensus control for discrete-time multi-agent systems with coding-decoding communication protocol, IEEE Transactions on Cybernetics, vol. 49, no. 12 , pp. $4335-4345,2019$

[41] S. Wen, Z. Zeng, T. Huang, Q. Meng, and W. Yao, Lag synchronization of switched neural networks via neural activation function and applications in image encryption, IEEE Transactions on Neural Networks and Learning Systems, vol. 26, no. 7, pp. 1493-1502, 2015.

[42] W. S. Wong and R. W. Brockett, Systems with finite communication bandwidth constraints - II: Stabilization with limited information feedback, IEEE Transactions on Automatic Control, vol. 44, no. 5, pp. 1049-1053, 1999.

[43] W. Wu, W. Zhou, and T. Chen, Cluster synchronization of linearly coupled complex networks under pinning control, IEEE Transactions on Circuits and Systems I: Regular Papers, vol. 56, no. 4, pp. 829-839, 2009.

[44] Y. Xu, J.Y. Li, R. Lu, C. Liu, and Y. Wu, Finite-horizon $l_{2}-l_{\infty}$ synchronization for time-varying Markovian jump neural networks under mixed-type attacks: Observer-based case, IEEE Transactions on Neural Networks and Learning Systems, vol. 30, no. 6, pp. 1695-1704, 2019.

[45] K. You and L. Xie, Minimum data rate for mean square stabilizability of linear systems with Markovian packet losses, IEEE Transactions on Automatic Control, vol. 56, no. 4, pp. 772-785, 2011.

[46] K. You and L. Xie, Network topology and communication data rate for consensusability of discrete-time multi-agent systems, IEEE Transactions on Automatic Control, vol. 56, no. 10, pp. 2262-2275, 2011.

[47] D. Yue and H. Li, Synchronization stability of continuous/discrete complex dynamical networks with interval time-varying delays, $\mathrm{Neu}$ rocomputing, vol. 73, no. 4-6, pp. 809-891, 2010.

[48] H. Zeng, J. H. Park, S.-P. Xiao, and Y. Liu, Further results on sampleddata control for master-slave synchronization of chaotic Lur'e systems with time delay, Nonlinear Dynamics, vol. 82, pp. 851-863, 2015.
[49] H. Zeng, K. L. Teo, Y. He, H. Xu and W. Wang, Sampled-data synchronization control for chaotic neural networks subject to actuator saturation, Neurocomputing, vol. 260, pp. 25-31, 2017.

[50] H. Zeng, Z.-L. Zhai, H. Yan and W. Wang, A new looped functional to synchronize neural networks with sampled-data control, IEEE Transactions on Neural Networks and Learning Systems, to be published. doi: 10.1109/TNNLS.2020.3027862.

[51] W. Zhang, C. Li, H. Li, and X. Yang, Cluster stochastic synchronization of complex dynamical networks via fixed-time control scheme, Neural Networks, vol. 124, pp. 12-19, 2020.

[52] Z. Zhao, Z. Wang, L. Zou and J. Guo, Set-membership filtering for time-varying complex networks with uniform quantisations over randomly delayed redundant channels, International Journal of Systems Science, vol. 51, no. 16, pp. 3364-3377, 2020.

[53] K. Zhu, J. Hu, Y. Liu, N. D. Alotaibi and F. E. Alsaadi, On $\ell_{2}-$ $\ell_{\infty}$ output-feedback control scheduled by stochastic communication protocol for two-dimensional switched systems, International Journal of Systems Science, in press, DOI: 10.1080/00207721.2021.1914768.

[54] L. Zou, Z. Wang, J. Hu, Y. Liu and X. Liu, Communication-protocolbased analysis and synthesis of networked systems: Progress, prospects and challenges, International Journal of Systems Science, in press, DOI: $10.1080 / 00207721.2021 .1917721$.

[55] L. Zou, Z. Wang, J. Hu and H. Dong, Ultimately bounded filtering subject to impulsive measurement outliers, IEEE Transactions on Automatic Control, in press, DOI: 10.1109/TAC.2021.3081256.

[56] L. Zou, Z. Wang, Q.-L. Han and D. H. Zhou, Full information estimation for time-varying systems subject to Round-Robin scheduling: A recursive filter approach, IEEE Transactions on Systems, Man, and Cybernetics: Systems, vol. 51, no. 3, pp. 1904-1916, 2021.

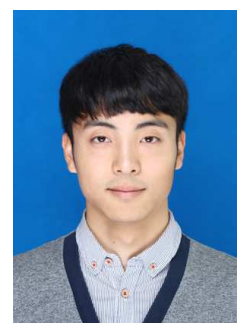

Jun-Yi Li was born in Henan province, China, in 1991. He received the M.S. degree in control science and engineering from Hangzhou Dianzi University, Hangzhou, China, in 2017. He is currently working toward the Ph.D. degree in Control Science and Engineering with the School of Automation, at Guangdong University of Technology, Guangzhou, China.

He was a visiting Ph.D. student with the Department of Computer Science, Brunel University London, Uxbridge, U.K., from October 2019 to October 2020. His research interests include complex dynamical networks, networked systems. 


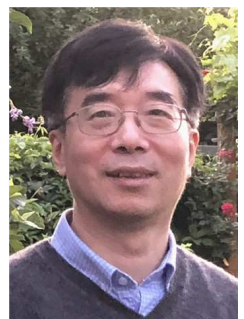

Zidong Wang (SM'03-F'14) was born in Jiangsu, China, in 1966. He received the B.Sc. degree in mathematics in 1986 from Suzhou University, Suzhou, China, and the M.Sc. degree in applied mathematics in 1990 and the Ph.D. degree in electrical engineering in 1994, both from Nanjing University of Science and Technology, Nanjing, China.

$\mathrm{He}$ is currently Professor of Dynamical Systems and Computing in the Department of Computer Science, Brunel University London, U.K. From 1990 to 2002, he held teaching and research appointments in universities in China, Germany and the UK. Prof. Wang's research interests include dynamical systems, signal processing, bioinformatics, control theory and applications. He has published more than 600 papers in international journals. He is a holder of the Alexander von Humboldt Research Fellowship of Germany, the JSPS Research Fellowship of Japan, William Mong Visiting Research Fellowship of Hong Kong.

Prof. Wang serves (or has served) as the Editor-in-Chief for International Journal of Systems Science, the Editor-in-Chief for Neurocomputing, the Editor-in-Chief for Systems Science \& Control Engineering, and an Associate Editor for 12 international journals including IEEE Transactions on Automatic Control, IEEE Transactions on Control Systems Technology, IEEE Transactions on Neural Networks, IEEE Transactions on Signal Processing, and IEEE Transactions on Systems, Man, and Cybernetics-Part C. He is a Member of the Academia Europaea, a Member of the European Academy of Sciences and Arts, an Academician of the International Academy for Systems and Cybernetic Sciences, a Fellow of the IEEE, a Fellow of the Royal Statistical Society and a member of program committee for many international conferences.

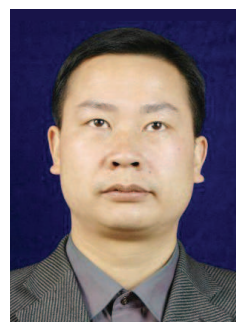

Renquan Lu (Senior Member, IEEE) received his $\mathrm{Ph} . \mathrm{D}$. degree in Control Science and Engineering from Zhejiang University, Hangzhou, China, in 2004. He was supported by the National Science Fund for Distinguished Young Scientists of China in 2014, honored as the Distinguished Professor of Pearl River Scholars Program of Guangdong Province, the Distinguished Professor of Yangtze River Scholars Program by the Ministry of Education of China in 2015 and 2017, respectively. Currently, he is a professor of the School of Automation at Guangdong University of Technology, Guangzhou, China.

His research interests include complex systems, networked control systems, and nonlinear systems.

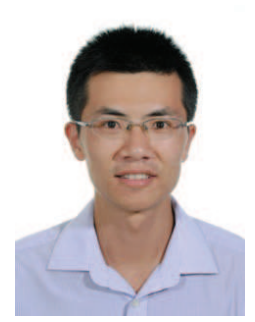

Yong Xu (Member, IEEE) was born in Zhejiang Province, China, in 1983. He received the B.S. degree in information engineering from Nanchang Hangkong University, Nanchang, China, in 2007, the M.S. degree in control science and engineering from Hangzhou Dianzi University, Hangzhou, China, in 2010, and the Ph.D. degree in control science and engineering from Zhejiang University, Hangzhou, China, in 2014. He was a visiting internship student with the department of Electronic and Computer Engineering, Hong Kong University of Science and Technology, Hong Kong, China, from June 2013 to November 2013, where he was a Research Fellow from February 2018 to August 2018. Now he is a professor with School of Automation, at Guangdong University of Technology, Guangzhou, China.

His research interests include PID control, networked control systems, state estimation, and positive systems. 